\title{
Group theory description of transformation pathway degeneracy during structural phase transformations
}

\author{
Yipeng $\mathrm{Gao}^{1}$, Rongpei $\mathrm{Shi}^{1}$, Jian-Feng $\mathrm{Nie}^{2}$, Suliman A. Dregia ${ }^{1}$, Yunzhi Wang ${ }^{1, *}$ \\ ${ }^{I}$ Department of Materials Science and Engineering, The Ohio State University, \\ Columbus, OH 43210, USA \\ ${ }^{2}$ Department of Materials Science and Engineering, Monash University, Clayton, \\ Victoria 3800, Australia
}

\begin{abstract}
Pathway degeneracy of structure transformations with symmetry breaking underpins the functionalities of a broad class of smart materials - the ferroics. Despite of its significance, there has been a lack of rigorous theoretical description of pathway degeneracy, leading to several case-dependent treatments which are not generally correct. In this work, we incorporate lattice correspondence into group theory to define and determine pathway degeneracy during structural transformations. In particular we show that a stabilizer can be determined by taking into account either the deformation relationship (under a given lattice correspondence) or the orientation relationship, through which deformation variant is defined rigorously and distinguished clearly from the orientation variant. Such a definition provides a theoretical foundation for investigating the formation of domain and defect structures arising from symmetry breaking during structural phase transformations.
\end{abstract}

Key words: structural phase transformation, crystal symmetry, pathway degeneracy, group theory, deformation variant 


\section{Introduction}

From the crystallographic point of view, symmetry breaking during structural transformations provides a natural means of achieving self-organized polydomain structures. These domains can switch from one to another by external fields such as stress, electrical and magnetic fields, offering special "smart" properties such as piezoelectricity, electro- and magneto-striction, superelasticity, shape-memory, and transformation toughening [1-5] to name a few. The symmetry breaking attending a phase transformation is understood on the basis of group theory [6-8], and the concept of orientation variants (i.e., the number of crystallographically equivalent domains of the low symmetry phase) has been established since 1970s [9, 10]. Such a group theory description of coexisting phases that are related by observed orientation relationships (ORs) has profound influences on the development of advanced geometrical theories of structures of hetero-phase interfaces such as the Coincidence Site Lattice theory [11] and the O-lattice theory [12]. However, such a group description applies to any two coexisting crystals, even if they were joined artificially without a transformation (e.g., epitaxial growth of one crystal on top of the other during vapor deposition). It is the number of crystallographically equivalent transformation pathways (TPs) for the structure change (i.e., the TP degeneracy or deformation variant) that determine the reversibility of the transformation and formation of various defect structures such as domain walls, twin boundaries and dislocations [13]. In order to describe TP degeneracy during a structural phase transformation, the fact that the two crystal structures are related by a deformation under a given correspondence before and after the transformation should be taken as an additional physical constraint besides the crystal structure information.

To illustrate the difference between orientation degeneracy and TP degeneracy at the intuitive level, we consider the relations between a square unit cell and a rectangular unit cell of two different lattices with a common origin. If the rectangle is oriented such that its long edge is parallel to an edge of the square, then two equivalent orientation variants are dictated by the symmetry and given OR. Likewise, if the long edge of the rectangle is made parallel to a diagonal of the square, there would be two equivalent variants of this OR. But in any other OR there would be four equivalent orientation variants of the rectangle relative to the square. An OR is defined whenever two crystals coexist, with or without contact. Now suppose a rectangle is made by stretching a square along one of its edges, i.e., with edge-to-edge correspondence. Clearly, there are two equivalent deformation pathways that satisfy the prescribed correspondence, leading to the so-called correspondence variant defined in martensitic 
transformation crystallography [2, 14]. Moreover, the number of correspondence variants is independent of any rotation of the rectangle relative to the square, i.e., it is independent of the OR for a fixed lattice correspondence (LC) [15-18]. It has been recognized [19] that the symmetry of the transformation strain, which includes the information of LC rather than OR, should be considered in the study of pathway degeneracy during martensitic transformations.

Thus the types of symmetry breaking and degeneracy are determined by the types of relationships between two crystals that one is considering, e.g., OR or LC. The former is characterized by rigid-body rotation and translation, leading respectively to crystallographically equivalent orientation variants and anti-phase domain variants, both of which have been well addressed in the literature [6,9]. The latter is characterized by a uniform lattice distortion and an internal atomic shuffle, which can also be described through the matching and splitting of Wyckoff position [7, 20-22]. Despite of its importance, several key issues in determining the latter degeneracy (referred to as TP degeneracy in this study) have not been realized and well-addressed in the literature, including: (1) besides the information of the symmetry groups of individual crystals involved during the transformation, a relationship connecting the two crystals of the parent and product phases is necessary and critical, which could be a piece of information characterizing the transformation pathway (e.g., LC); (2) the two symmetry groups of individual crystals cannot be used directly in Lagrange's Theorem, because it is not guaranteed that there is a group-subgroup relationship between them; instead, a stabilizer (or intersection group) has to be determined first, which is strictly a subgroup of the symmetry group of the parent phase and satisfies the requirement of Lagrange's Theorem; (3) the effects of shuffle on TP degeneracy should be included within the framework of group theory. Overlook of the above issues may lead to case-dependent treatments which are not generally correct. For example, to exclude the effect of orientation dependence, one popular way to determine TP degeneracy is by using proper symmetry operations only, which is workable in some shape memory alloy systems [15]. However, such a treatment is limited not only because the degeneracy caused by breaking of mirror symmetry cannot be captured, but also it fails when dealing with reconstructive martensitic transformations [13]. One classical counter-example is the face-centered cubic (FCC) to body-centered cubic (BCC) transformation through the Bain path in pure iron. The point group of both FCC and BCC lattices are $\mathbf{m} \overline{3} \mathbf{m}$, leading to only one pathway according to this treatment, which is definitely contradict to the three equivalent Bain pathways intuitively well-known in the literature [16-19, 23]. 
TP degeneracy plays a critical role in formulating theoretical descriptions of structural phase transformations, as has been demonstrated in the phenomenological theory of martensitic crystallography (PTMC) [16-18] and Landau theory of phase transformations [24]. Because of the lack of rigorous group theory description of the TP degeneracy, however, orientation degeneracy determined by the group theory formulation [9] using a particular OR as the constraint has been used frequently in literature as the TP degeneracy [25, 26]. As has been discussed above, however, the TP degeneracy should be determined by the constraint of LC and be independent of OR. Consider again the classical FCC to BCC transformation in steels. If the Kurdyumov-Sachs and Nishiyama-Wasserman ORs commonly observed in the experiments are used, then the number of orientation variants will be 24 and 12, respectively. Only when the Baker-Nutting OR or Bain OR (not Bain LC) is used, the number of orientation degeneracy equals the number of TP degeneracy, which is 3 . Note that the Baker-Nutting OR has not been observed in experiments. Another well-known example is the BCC to hexagonal-close-packed (HCP) structural transformation in Ti-based and Zr-based alloys. Depending on the experimentally observed ORs, including the Burgers, Potter, Pistch-Schrader and Rong-Dunlp [27], the number of orientation variants can be systematically obtained through group theory [9]. However, the TP degeneracy for the Burgers path has yet been determined rigorously, because of the difficulty caused by the internal atomic shuffle during the transformation.

In this work, we formulate a group theory framework to define and determine TP degeneracy during structural transformations that involve a lattice deformation. Through a change of basis, a stabilizer can be determined by either LC or OR, leading to rigorous definitions of TP degeneracy and orientation degeneracy within a general framework. Such a definition of TP degeneracy is critical in formulating Landau free energy dictated by the symmetry of Hamiltonian and investigating deformation mechanisms that governs microstructural evolution and materials properties during structural transformations. Several examples are presented, through which not only the standardized mathematical procedure to determine the degeneracies but also the symmetry related features in terms of microstructures are described in great details.

\section{Mathematical formulations of orientation variant and deformation variant}

We consider an $\alpha \rightarrow \beta$ phase transformation, where the two crystals have point groups $\mathbf{H}$ and $\mathbf{H}$, respectively. Individual operations in the two groups, $\mathbf{h}^{\alpha} \in \mathbf{H}^{\alpha}$ and 
$\mathbf{h}^{\beta} \in \mathbf{H}^{\beta}$, can be represented in matrices in particular coordinates. Symmetry dictated degeneracy during the transformation is given by the following relation $[9,10]$ :

$$
N_{\alpha \rightarrow \beta}=\frac{\left|\mathbf{H}^{\alpha}\right|}{\left|\mathbf{J}^{\alpha / \beta}\right|}
$$

where $|\mathbf{H}|$ is the order of the group of the parent phase, and $\mathbf{J}^{\alpha / \beta}$ is known as the stabilizer subgroup, it consists of symmetry operations that are preserved during the transformation. Eqn. (1) follows from the coset decomposition of a group ( $\mathbf{H}$ ) in terms of its subgroup $\left(\mathbf{J}^{\alpha / \beta}\right)$, and the quotient (called the index of $\mathbf{J}^{\alpha / \beta}$ in $\mathbf{H}^{\alpha}$ ) is guaranteed to be an integer by Lagrange's Theorem [8]. In some particular phase transformations (e.g., ferroic phase transitions) $\mathbf{J}^{\alpha / \beta}=\mathbf{H}^{\beta}$, which allows Eqn. (1) to be expressed as the ratio of the orders of the groups of the two phases (i.e., $\left|\mathbf{H}^{\alpha}\right| /\left|\mathbf{H}^{\beta}\right|$ ). However, such an expression not only obscures the fact that using $\mathbf{H}^{\beta}$ in the denominator is only conditionally correct, but also overlooks the dependence of the degeneracy on a critical link (e.g., OR or LC) between the two groups. As pointed out by Cahn and Kalonji [9], the orientation degeneracy should depend on the stabilizer (or intersection group) dictated by the OR between two crystals, and parallel conclusion could also be expected for TP degeneracy. In general, the stabilizer is a subgroup of both $\mathbf{H}$ and $\mathbf{H}$, and it consists of operations that are common to the two groups, not belonging to either group exclusively $[9,10]$.

Considering the acrystal defined in the $B_{\alpha}$ basis, $\mathbf{H}$ can be represented in $B_{\alpha}$ as $\mathbf{H}_{\alpha}^{\alpha}$ (similarly, $\mathbf{H}$ are represented in $B_{\beta}$ as $\mathbf{H}_{\beta}^{\beta}$ ). $B_{\alpha}=\left\{\mathbf{a}_{i}^{\alpha}\right\}$ and $B_{\beta}=\left\{\mathbf{a}_{i}^{\beta}\right\}$, with $i=$ 1,2,3. In order to calculate their intersection group, $\mathbf{H}$ and $\mathbf{H}$ should be represented in the same basis, and an operator, $\hat{\mathbf{T}}$, can be used to link $B_{\alpha}$ and $B_{\beta}$ without loss of generality. The superscript used here indicates the crystal lattice ( $\alpha$ or $\beta$ ) that the vector (or operation) belongs to. Under the action of a point operation $\mathbf{h}^{\alpha}$, the $\alpha$ crystal will be self-coincident, but every vector will be transformed to a new position

$$
\mathbf{S}^{\alpha}=\mathbf{h}^{\alpha} \mathbf{r}^{\alpha}
$$


where $\mathbf{r}^{\alpha}$ and $\mathbf{s}^{\alpha}$ are two vectors in $\alpha$ crystal that are related by the symmetry operation $\mathbf{h}^{\alpha}$. Likewise, under the action of $\mathbf{h}^{\beta}$, the $\beta$ crystal will be self-coincident,

$$
\mathbf{s}^{\beta}=\mathbf{h}^{\beta} \mathbf{r}^{\beta}
$$

And the stabilizer group is determined as the following,

$$
\mathbf{J}^{\alpha / \beta}=\left\{\mathbf{h}^{\alpha}: \mathbf{h}^{\alpha}=\hat{\mathbf{T}}^{-1} \mathbf{h}^{\beta} \hat{\mathbf{T}}, \mathbf{h}^{\alpha} \in \mathbf{H}^{\alpha}, \mathbf{h}^{\beta} \in \mathbf{H}^{\beta}\right\}
$$

The stabilizer is made up of operations that are common to the two groups; hence, their representation matrices are identical when referred to the same basis (regardless of which basis is used for reference). The algebraic relations leading to Eqn. (3) are summarized by the circuit diagram in Fig. 1, where the top and bottom legs correspond to the transformation through operator $\hat{\mathbf{T}}$, and the left and right legs corresponds to the symmetry operations. According to Eqns. (1)\&(3), symmetry dictated degeneracy should be determined by $\mathbf{H}, \mathbf{H}$ and $\hat{\mathbf{T}}$. Note that $\mathbf{H}$ and H only provide the symmetry information of involved crystals without any concern about the relationship between them. Critical information linking the two crystals are given by the generic operator $\hat{\mathbf{T}}$ in Fig. 1(a), and different choice of $\hat{\mathbf{T}}$ could lead to different degeneracy. In the followings, different definitions of $\hat{\mathbf{T}}$ associated with different kinds of variants will be used, which represent either change of basis, lattice distortion, or the combination of the two.

\subsection{Orientation Variants}

In order to compare and contrast orientation degeneracy with the TP degeneracy, we focus on developing the stabilizer groups for the two cases. For a pair of crystals, the

OR is expressed by a change-of-basis (passive) operator between $B_{\alpha}$ and $B_{\beta}$, $\hat{\mathbf{P}}$, and the coordinate transformation is expressed in matrix form as

$$
\mathbf{r}_{\beta}=\mathbf{P r}_{\alpha}
$$

where $\mathbf{r}_{\alpha}$ and $\mathbf{r}_{\beta}$ are the same vector $\mathbf{r}$ relative to the two bases of $B_{\alpha}$ and $B_{\beta}$ (the top leg in Fig. 1(b)) as indicated by the subscript. Since we seek operations that preserve the OR, we require the $\hat{\mathbf{P}}$ operator to remain invariant, so that (the bottom leg in Fig. 
$1(b))$,

$$
\mathbf{s}_{\beta}=\mathbf{P s}_{\alpha}
$$

and we obtain the condition for OR invariance.

$$
\begin{aligned}
& \mathbf{J}_{O}^{\alpha / \beta}=\left\{\mathbf{h}^{\alpha}: \mathbf{h}_{\alpha}^{\alpha}=\mathbf{P}^{-1} \mathbf{h}_{\beta}^{\beta} \mathbf{P}, \mathbf{h}^{\alpha} \in \mathbf{H}^{\alpha}, \mathbf{h}^{\beta} \in \mathbf{H}^{\beta}\right\} \\
& \operatorname{or} \mathbf{J}_{O}^{\alpha / \beta}=\left\{\mathbf{h}^{\alpha}: \mathbf{h}_{\alpha}^{\alpha}=\mathbf{h}_{\alpha}^{\beta}, \mathbf{h}^{\alpha} \in \mathbf{H}^{\alpha}, \mathbf{h}^{\beta} \in \mathbf{H}^{\beta}\right\}
\end{aligned}
$$

The OR stabilizer is made up of operations that are common to the two coexisting crystals; hence, their representation matrices are identical when referred to the same basis (regardless of which basis is used for reference). In this case, critical information are given by the operator $\hat{\mathbf{P}}$, which characterizes the spatial relation between the two bases of $B_{\alpha}$ and $B_{\beta}$. Such a relation requires the coexistence of parent and product crystals, but does not require the deformation path between them. Eqn. (5a) can be derived from the generic equation of Eqn. (3) when $\hat{\mathbf{T}}$ is chosen as $\hat{\mathbf{P}}$.

Since $\hat{\mathbf{P}}$ is purely a change of bases, all the vectors should be referred to certain basis, as indicated by the subscript in Fig. 1(b).

\subsection{Deformation Variants}

Since the existing concept of correspondence variant does not describe precisely pathway degeneracy when internal shuffle exists in addition to a homogeneous deformation, a new concept of deformation variant is introduced to capture the pathway degeneracy and avoid possible terminology confusion. We assume that the $\alpha \rightarrow \beta$ phase transformation is accomplished by a deformation so that every vector in the $\alpha$ crystal, $\mathbf{r}^{\alpha}$, is deformed into a corresponding vector in the $\beta$ crystal, $\mathbf{r}^{\beta}$, and we have

$$
\mathbf{r}_{\alpha}^{\beta}=\mathbf{A} \mathbf{r}_{\alpha}^{\alpha}+\boldsymbol{\sigma}_{\alpha}
$$

which shows the both the homogeneous deformation described matrix $\mathbf{A}$ and the shuffle vector $\boldsymbol{\sigma}$. In general, $\boldsymbol{\sigma}$ is a periodic function of spatial coordinates. Note that $\hat{\mathbf{A}}$ is an active operator describing deformation between two crystals, which is fundamentally different from the passive operator $\hat{\mathbf{P}}$ introduced in the OR case. The 
homogeneous deformation $\hat{\mathbf{A}}$ is uniquely defined by an assumed correspondence between a triplet of lattice vectors before and after the deformation. First of all, we consider lattice distortion described in the same basis (i.e., $B_{\alpha}$ ) for simplicity, then the $\hat{\mathbf{T}}$ operator can be interpreted as,

$$
\mathbf{r}_{\alpha}^{\beta}=\hat{\mathbf{T}} \mathbf{r}_{\alpha}^{\alpha} \Rightarrow \mathbf{r}_{\alpha}^{\beta}=\mathbf{A} \mathbf{r}_{\alpha}^{\alpha}+\boldsymbol{\sigma}_{\alpha}
$$

And similarly,

$$
\mathbf{s}_{\alpha}^{\beta}=\hat{\mathbf{T}} \mathbf{s}_{\alpha}^{\alpha} \Rightarrow \mathbf{s}_{\alpha}^{\beta}=\mathbf{A} \mathbf{s}_{\alpha}^{\alpha^{\prime}}+\boldsymbol{\sigma}_{\alpha}
$$

where Eqns. (7) and (8) are illustrated by the top and bottom legs in Fig. 1(c).

Since we seek operations that preserve the LC, we require the $\hat{\mathbf{T}}$ operator to remain invariant. In other words, the same results of $\mathbf{s}_{\alpha}^{\beta}$ from $\mathbf{r}_{\alpha}^{\alpha}$ should be reached when going through the top/right legs and the left/bottom legs.

For the top and right legs,

$$
\mathbf{s}_{\alpha}^{\beta}=\mathbf{h}_{\alpha}^{\beta} \mathbf{r}_{\alpha}^{\beta}=\mathbf{h}_{\alpha}^{\beta}\left(\mathbf{A r}_{\alpha}^{\alpha}+\boldsymbol{\sigma}_{\alpha}\right)
$$

For the left and bottom legs,

$$
\mathbf{s}_{\alpha}^{\beta}=\mathbf{A} \mathbf{s}_{\alpha}^{\alpha}+\boldsymbol{\sigma}_{\alpha}=\mathbf{A} \mathbf{h}_{\alpha}^{\alpha} \mathbf{r}_{\alpha}^{\alpha}+\boldsymbol{\sigma}_{\alpha}
$$

Comparing Eqns. (9a) and (9b), we have

$$
\mathbf{h}_{\alpha}^{\beta} \mathbf{A} \mathbf{r}_{\alpha}^{\alpha}+\mathbf{h}_{\alpha}^{\beta} \boldsymbol{\sigma}_{\alpha}=\mathbf{A} \mathbf{h}_{\alpha}^{\alpha} \mathbf{r}_{\alpha}^{\alpha}+\boldsymbol{\sigma}_{\alpha}
$$

Because the above equation should be satisfied for any lattice vector $\mathbf{r}_{\alpha}^{\alpha}$, it requires

$$
\left\{\begin{array}{c}
\mathbf{h}_{\alpha}^{\beta} \mathbf{A}=\mathbf{A} \mathbf{h}_{\alpha}^{\alpha} \\
\mathbf{h}_{\alpha}^{\beta} \boldsymbol{\sigma}_{\alpha}=\boldsymbol{\sigma}_{\alpha}
\end{array}\right.
$$

As a result, the stabilizer that satisfies the condition for LC invariance is,

$$
\mathbf{J}_{D}^{\alpha / \beta}=\left\{\begin{array}{l}
\mathbf{h}^{\alpha}: \mathbf{h}_{\alpha}^{\alpha}=\mathbf{A}^{-1} \mathbf{h}_{\alpha}^{\beta} \mathbf{A}, \mathbf{h}_{\alpha}^{\beta} \boldsymbol{\sigma}_{\alpha}=\boldsymbol{\sigma}_{\alpha}, \\
\mathbf{h}^{\alpha} \in \mathbf{H}^{\alpha}, \mathbf{h}^{\beta} \in \mathbf{H}^{\beta}
\end{array}\right\}
$$

Eqn. (12) can be derived from the generic equation of Eqn. (3) when $\hat{\mathbf{T}}$ is a lattice distortion. Since the lattice distortion is described entirely in the $B_{\alpha}$ basis, all the 
vectors are referred to $B_{\alpha}$ in Fig. 1(c). Note that Fig. 1(b) and Fig. 1(c) are two distinctive ways to realize Fig. 1(a) by choosing different kinds of $\hat{\mathbf{T}}$. In the case of Fig. 1(b), the operations of $\mathbf{h}^{\alpha}$ and $\mathbf{h}^{\beta}$ exert on the same vector in different bases (i.e., $\mathbf{h}_{\alpha}^{\alpha}$ on $\mathbf{r}_{\alpha}, \mathbf{h}_{\beta}^{\beta}$ on $\mathbf{r}_{\beta}$ ). In contrast, the operations of $\mathbf{h}^{\alpha}$ and $\mathbf{h}^{\beta}$ exert on different vectors in the same basis in Fig. 1(c) (i.e., $\mathbf{h}_{\alpha}^{\alpha}$ on $\mathbf{r}_{\alpha}^{\alpha}, \mathbf{h}_{\alpha}^{\beta}$ on $\mathbf{r}_{\alpha}^{\beta}$ ). However, the $\hat{\mathbf{T}}$ operator in Fig. 1(c) includes both LC and OR information, which may conceal the fact that the TP degeneracy should not depend on OR. In the followings, we will show that the TP degeneracy is purely determined by LC rather than OR. In addition, the generic operator $\hat{\mathbf{T}}$ could also be chosen as a transformation including both change of basis and lattice distortion, which will lead to a general formulation for the determination of transformation related degeneracies through standardized mathematical procedures.

We could consider the correspondence between primitive bases of the two crystals $B_{\alpha} \rightarrow B_{\beta}$ (here $B_{\beta}$ is a particular $\beta$-basis, it is the image of $B_{\alpha}$ under the deformation, i.e. $\mathbf{a}_{i}^{\alpha} \rightarrow \mathbf{a}_{i}^{\beta}, i=1,2,3$ ). Each lattice vector (axis) in the $\alpha$-lattice is mapped to a unique lattice vector (axis) in $\beta$, and the two corresponding vectors are not necessarily parallel. Moreover, since the change-of-basis operator $\hat{\mathbf{P}}$ from $B_{\alpha}$ to $B_{\beta}$ is $\mathbf{P}=\mathbf{A}^{-1}$, the matrices that represent corresponding vectors are identical, $\mathbf{r}_{\beta}^{\beta}=\mathbf{r}_{\alpha}^{\alpha}$.Also, we have $\boldsymbol{\sigma}_{\beta}=\mathbf{P} \boldsymbol{\sigma}_{\alpha}=\mathbf{A}^{-1} \boldsymbol{\sigma}_{\alpha}$. As a result, Eqn. (12) can be rewritten as,

$$
\mathbf{J}_{D}^{\alpha / \beta}=\left\{\begin{array}{l}
\mathbf{h}^{\alpha}: \mathbf{h}_{\alpha}^{\alpha}=\mathbf{h}_{\beta}^{\beta}, \mathbf{h}_{\beta}^{\beta} \boldsymbol{\sigma}_{\beta}=\boldsymbol{\sigma}_{\beta}, \\
\mathbf{h}^{\alpha} \in \mathbf{H}^{\alpha}, \mathbf{h}^{\beta} \in \mathbf{H}^{\beta}
\end{array}\right\}
$$

Above equation can be simplified if the shuffle vector is also zero, which suggests a homogeneous deformation (See appendix),

$$
\mathbf{J}_{D}^{\alpha / \beta}=\left\{\mathbf{h}^{\alpha}: \mathbf{h}_{\alpha}^{\alpha}=\mathbf{h}_{\beta}^{\beta}, \mathbf{h}^{\alpha} \in \mathbf{H}^{\alpha}, \mathbf{h}^{\beta} \in \mathbf{H}^{\beta}\right\}
$$

Here, the operations of interest are conjugated by the homogeneous deformation, as opposed to being identical for OR variants. The equality of matrix representations in the two corresponding bases means that the two operations have similar actions on corresponding vectors, as they map corresponding vectors into corresponding vectors, i.e., they preserve the correspondence. In the case of orientation variant, the 
operations of interest map parallel vectors into parallel vectors, i.e., they preserve the parallelism. Eqn. (14) also reveals that the deformation stabilizer is unchanged by a change in OR because the matrices of type $\mathbf{h}_{\beta}^{\beta}$ are invariant under a rigid-body rotation of $B_{\beta}$. Note that $B_{\beta}$ is a special basis that leaves $\mathbf{T}=\mathbf{P A}=\mathbf{I}$ (See appendix Fig. A-1(a)), and the $\hat{\mathbf{T}}$ operator here can be regarded as a general transformation including both lattice distortion and the change of basis from $B_{\alpha}$ to $B_{\beta}$. In the above formulations, however, the form of $\hat{\mathbf{T}}$ is simplified (as identity) while the form of $\mathbf{h}^{\alpha}$ and $\mathbf{h}^{\beta}$ are complicated, which may not be convenient for a standardized mathematical procedure.

In fact, the symmetry operations in $\beta$ crystal may not be easily represented in $B_{\beta}$, and a general basis $B_{\beta^{*}}$ (rather than a special basis of $B_{\beta}$ ) should be considered, and the $\hat{\mathbf{T}}$ operator, that links $\mathbf{r}_{\alpha}^{\alpha}$ and $\mathbf{r}_{\beta^{*}}^{\beta}$, can be interpreted as,

$$
\mathbf{r}_{\beta^{*}}^{\beta}=\hat{\mathbf{T}} \mathbf{r}_{\alpha}^{\alpha} \Rightarrow \mathbf{r}_{\beta^{*}}^{\beta}=\operatorname{Pr}_{\alpha}^{\beta}=\mathbf{P}\left(\mathrm{Ar}_{\alpha}^{\alpha}+\boldsymbol{\sigma}_{\alpha}\right)
$$

And similarly,

$$
\mathbf{s}_{\beta^{*}}^{\beta{ }^{\prime}}=\hat{\mathbf{T}}_{\alpha}^{\alpha{ }^{\prime}} \Rightarrow \mathbf{s}_{\beta^{*}}^{\beta{ }^{\prime}}=\mathbf{P} \mathbf{s}_{\alpha}^{\beta}=\mathbf{P}\left(\mathbf{A} \mathbf{s}_{\alpha}^{\alpha{ }^{\prime}+\boldsymbol{\sigma}_{\alpha}}\right)
$$

where Eqns. (15a) and (15b) are illustrated by the top and bottom legs (See appendix Fig. A-1(a)).

Parallel to Eqn. (14), the stabilizer represented in the general basis of $B_{\beta^{*}}$ can be written as follows when the shuffle vector is also zero (See appendix Fig. A-2(b)),

$$
\mathbf{J}_{D}^{\alpha / \beta}=\left\{\mathbf{h}^{\alpha}: \mathbf{h}_{\alpha}^{\alpha}=\mathbf{C}^{-1} \mathbf{h}_{\beta^{*}}^{\beta} \mathbf{C}, \mathbf{h}^{\alpha} \in \mathbf{H}^{\alpha}, \mathbf{h}^{\beta} \in \mathbf{H}^{\beta}\right\}
$$

Where $\mathbf{C}=\mathbf{P A}$ can be considered as a general change of basis from $B_{\alpha}$ to $B_{\beta}$, which is the so-called correspondence matrix in martensitic crystallography [15-17]. Note that $\mathbf{C}$ is an operator including the information of LC only (no OR information).

Comparing Eqn. (3) with Eqns. (5) \& (16), it can be found that $\hat{\mathbf{T}}=\hat{\mathbf{P}}$ for orientation variant, while $\hat{\mathbf{T}}=\hat{\mathbf{C}}$ for deformation variant. In the former, $\hat{\mathbf{T}}$ characterizes the 
orientation relationship (i.e., alignment between vectors), while in the latter, $\hat{\mathbf{T}}$ characterizes the deformation relationship (i.e., correspondence between vectors). In these two cases, critical information is provided by the operator $\hat{\mathbf{T}}$, through which deformation variant and orientation variant are distinguished. Note that $\hat{\mathbf{T}}$ does not include any information about crystal symmetry, but it provides a linkage between two symmetry groups ( $\mathbf{H}$ and $\mathbf{H}$ ). Taking advantage of such a description, a systematic framework to formulate symmetry dictated degeneracy could be developed. Different kinds of degeneracy can be determined by the same equation, but with different choices of operator $\hat{\mathbf{T}}$. Besides pathway degeneracy and orientation degeneracy, the degeneracy caused by the break of chiral symmetry could also be rigorously defined in this framework, which is investigated broadly in stereochemistry and plays an important role in some ferroic materials.

\section{Determination of transformation pathway degeneracy - examples of applications}

Symmetry dictated degeneracies are typical for structural transformations among FCC, BCC and HCP crystals. In particular, the orientation degeneracies during these transformations have been well documented in the literature [28] according to experimentally observed ORs. However, the pathway (or deformation) degeneracies of these transformations have not been rigorously determined based on group theory. Note that HCP is fundamentally different from FCC and BCC because it includes a two-atom motif in each lattice point. As a result, internal shuffle has to be involved during structural transformations involving an HCP phase, leading to additional degeneracy if symmetry is broken by the shuffle. Problems are met when dealing with the BCC to HCP (e.g., Ti-based and Zr-based alloys) and the FCC to HCP (e.g., Co-based and Fe-Mn-Si-based alloys) transformations, in both of which additional symmetry breaking can arise from internal shuffles besides homogeneous deformation [28-31]. Furthermore, even though the point group of HCP is known as 6/mmm, which is deduced from its space group of $\mathrm{P}_{3} / \mathrm{mmc}$ (also called the point group of its space group), no occupied Wyckoff position has site symmetry as high as 6/mmm in HCP. As a result, the general formulation for TP degeneracy (Eqn. (12) or (13)) should be used, which will be further discussed in Section 3.3.

Applying the above group theory framework, one can derive a complete set of TP 
degeneracies for transformations among FCC, BCC and HCP, as shown in Fig. 2 where all the groups for degeneracy determination (including symmetry groups of FCC/BCC/HCP and the stabilizers between each pair of them) are given for the following LCs:

$$
\begin{gathered}
{[100]_{F C C} \leftrightarrow[110]_{B C C} \leftrightarrow[1 \overline{1} 00]_{H C P}} \\
{[101]_{F C C} \leftrightarrow[111]_{B C C} \leftrightarrow \frac{2}{3}[2 \overline{1} \overline{1} 0]_{H C P},} \\
{[0 \overline{1} 0]_{F C C} \leftrightarrow[1 \overline{1} 0]_{B C C} \leftrightarrow[0001]_{H C P}}
\end{gathered}
$$

In the above relationships, the LC between FCC and BCC is the Bain correspondence and that between BCC and HCP is the Burgers correspondence. Due to the transitive feature of LCs, the correspondence between FCC and HCP is fixed. Based on the above description, the transformation mechanism between FCC and HCP can be interpreted as a homogeneous lattice distortion with additional internal shuffle in every two atomic layers, which is equivalent to the process of converting an FCC structure to an HCP structure through the passage of a Shockley partial dislocation in every two layers of $\{111\}_{F C C}[30-32]$. Furthermore, the pathway degeneracy is doubled due to symmetry breaking caused by internal shuffle during the BCC to HCP (and FCC to $\mathrm{HCP}$ ) transformation, but it does not change during the reversal transformations (i.e., $\mathrm{HCP}$ to $\mathrm{BCC}, \mathrm{HCP}$ to $\mathrm{FCC}$ ), which can be readily captured in our approach.

Pathway degeneracy is critical for the functionality of ferroic smart materials. In order to achieve good reversibility in shape memory alloys, pathway degeneracy of either forward or backward transformation has to be 1. If path degeneracies for both forward and backward transformations are larger than 1, it is inevitably irreversible and defect structures are induced by the transformations [13]. Such a mechanism is evidenced by the slip martensite substructure observed in low-carbon steels [33]. On the other hand, piezoelectricity or magnetostriction in ferroelectrics or ferromagnetic could be enhanced greatly by domain switching through multiple TPs near the so-called morphotropic phase boundary [4], where a tetragonal to rhombohedral transformation is involved, and the path degeneracies for forward and backward transformations are 4 and 3, respectively. The multiplicities of pathways in both transformation directions are necessary to achieve enhanced properties.

Since the number of deformation variants or TPs is independent of OR and so does 
the Landau free energy that characterizes the phase transformations, it is the deformation variants rather than orientation variants should be used as the order parameter in the Landau free energy. Because of the nature of the OR-independence of the deformation variants, the transformation can be described by an energy landscape defined in a six-dimensional strain space instead of a general nine-dimensional space, which is a significant simplification for the Landau free energy formulations [34, 35]. For example, eigenstrains (or their functions) are usually chosen as the order parameters in Landau theory of MTs and eigenstrain is orientation independent, which reduces the degrees of freedom of the system and simplifies the free energy formulation. In literature, the terminology of "orientation variants" rather than "deformation variants" have been used frequently in the Landau free energy as the order parameters, and the number of order parameters are determined ambiguously by either orientation degeneracy or TP degeneracy [36-39]. Not only could such a treatment lead to incorrect microstructural degeneracy (such as the number of crystallographically equivalent habit planes or multi-variant domain patterns), but also it conceals the fact that TP degeneracy described by the Landau free energy should be independent of OR.

In general, besides crystal symmetry and lattice parameters of the parent and product phases, LC needs to be introduced as an additional constraint into the group theory description of structural transformations, by which the crystal structure change and symmetry breaking can be captured. Only with a proper account of the symmetry reduction can phenomenological models be established to predict self-accommodating morphological patterns, crystallographic features and defects generated by the transformations.

In order to show determination of TP degeneracy and illustrate the importance of using deformation variant rather than orientation variant in Landau theory of phase transformations, two examples are presented below. The first one is a generic 2D example, through which the standardized mathematical procedure to determine symmetry-related degeneracies is illustrated. The second one is an engineering problem regarding how the degeneracy influences the symmetry-dictated features of microstructures.

\subsection{Primitive Rectangular $\rightarrow$ Centered Rectangular Transformation (2D)}

As shown in Fig. 3, the lattice transforms by a uniform deformation (simple shear) from a primitive-rectangular phase $\alpha$ (point group: $2 \mathrm{~mm}$ ) to a centered-rectangular 
phase $\beta$ (point group: $2 \mathrm{~mm}$ ) with OR $[10]_{\alpha} \|[10]_{\beta}$. By using the lattice parameters indicated in Fig. 3, a lattice correspondence can be derived: $[10]_{\alpha} \rightarrow[10]_{\beta}$ and $[01]_{\alpha} \rightarrow 1 / 2[11]_{\beta}$. According to the method developed above, there is one orientation variant (stabilizer: $2 \mathrm{~mm}$ ) and two deformation variants (stabilizer: 2) during this transformation.

Relative to the bases $B_{\alpha}$ and $B_{\beta^{*}}$ indicated in Fig. 3, the point groups for both the parent phase and product phase are $2 \mathrm{~mm}$, which can be represented in their respective bases as follows,

$$
\begin{aligned}
\mathbf{H}_{\alpha}^{\alpha} & =\left\{\left[\begin{array}{ll}
1 & 0 \\
0 & 1
\end{array}\right],\left[\begin{array}{cc}
-1 & 0 \\
0 & -1
\end{array}\right],\left[\begin{array}{cc}
1 & 0 \\
0 & -1
\end{array}\right],\left[\begin{array}{cc}
-1 & 0 \\
0 & 1
\end{array}\right]\right\} \\
\mathbf{H}_{\beta^{*}}^{\beta} & =\left\{\left[\begin{array}{ll}
1 & 0 \\
0 & 1
\end{array}\right],\left[\begin{array}{cc}
-1 & 0 \\
0 & -1
\end{array}\right],\left[\begin{array}{cc}
1 & 0 \\
0 & -1
\end{array}\right],\left[\begin{array}{cc}
-1 & 0 \\
0 & 1
\end{array}\right]\right\}
\end{aligned}
$$

For the orientation relationship with $[10]_{\alpha} \|[10]_{\beta}$, and with lattice parameters indicated, the operator $\mathbf{P}$ equals:

$$
\mathbf{P}=\left[\begin{array}{ll}
1 & 0 \\
0 & 1
\end{array}\right]
$$

And $\mathbf{H}^{\beta}$ can be represented in $B_{\alpha}$ through the change-of-basis matrix $\mathbf{P}$ :

$$
\mathbf{H}_{\alpha}^{\beta}(O)=\mathbf{P}^{-1} \mathbf{H}_{\beta^{*}}^{\beta} \mathbf{P}=\left\{\left[\begin{array}{cc}
1 & 0 \\
0 & 1
\end{array}\right],\left[\begin{array}{cc}
-1 & 0 \\
0 & -1
\end{array}\right],\left[\begin{array}{cc}
1 & 0 \\
0 & -1
\end{array}\right],\left[\begin{array}{cc}
-1 & 0 \\
0 & 1
\end{array}\right]\right\}
$$

The stabilizer for the OR is:

$$
\mathbf{J}_{O}^{P R / C R}=\left\{\left[\begin{array}{cc}
1 & 0 \\
0 & 1
\end{array}\right],\left[\begin{array}{cc}
-1 & 0 \\
0 & -1
\end{array}\right],\left[\begin{array}{cc}
1 & 0 \\
0 & -1
\end{array}\right],\left[\begin{array}{cc}
-1 & 0 \\
0 & 1
\end{array}\right]\right\}
$$

And clearly the stabilizer is $2 \mathrm{~mm}$. Thus, there is only one orientation variant determined by Eqn. (1).

On the other hand, based on Fig. 3, a lattice correspondence (LC) can be derived as: $[10]_{\alpha} \rightarrow[10]_{\beta} ;[01]_{\alpha} \rightarrow 1 / 2[11]_{\beta}$.

The operator $\mathbf{A}$ and $\mathbf{C}$ are realized as follows

$$
\begin{aligned}
& \mathbf{A}=\left[\begin{array}{cc}
1 & 0.5 \\
0 & 1
\end{array}\right] \\
& \mathbf{C}=\mathbf{P A}=\left[\begin{array}{cc}
1 & 0.5 \\
0 & 1
\end{array}\right]
\end{aligned}
$$


Thus, $\mathbf{H}^{\beta}$ can be represented in $B_{\beta *}$ as:

$$
\mathbf{H}_{\alpha}^{\beta}(D)=\mathbf{C}^{-1} \mathbf{H}_{\beta^{*}}^{\beta} \mathbf{C}=\left\{\left[\begin{array}{cc}
1 & 0 \\
0 & 1
\end{array}\right],\left[\begin{array}{cc}
-1 & 0 \\
0 & -1
\end{array}\right],\left[\begin{array}{cc}
1 & 1 \\
0 & -1
\end{array}\right],\left[\begin{array}{cc}
-1 & -1 \\
0 & 1
\end{array}\right]\right\}
$$

The stabilizer for deformation variant is given by Eqn. (14),

$$
\mathbf{J}_{D}^{P R / C R}=\left\{\left[\begin{array}{ll}
1 & 0 \\
0 & 1
\end{array}\right],\left[\begin{array}{cc}
-1 & 0 \\
0 & -1
\end{array}\right]\right\}
$$

Thus, the stabilizer subgroup is 2, and according to Eqn. (1), there are two deformation variants.

In general, the mathematical procedure to determine the numbers of orientation variants and deformation variants follows 5 steps: (i) writing down the symmetry groups for the parent and product phases in respective bases $\left(\mathbf{H}_{\alpha}^{\alpha}\right.$ and $\left.\mathbf{H}_{\beta^{*}}^{\beta}\right)$; (ii) determining the relationship between the two bases $\left(B_{\alpha}\right.$ and $\left.B_{\beta *}\right)$ that is described by $\hat{\mathbf{T}}(\hat{\mathbf{T}}=\hat{\mathbf{P}}$ for orientation variant, $\hat{\mathbf{T}}=\hat{\mathbf{C}}$ for deformation variant); (iii) representing $\mathbf{H}_{\beta^{*}}^{\beta}$ in $B_{\alpha}\left(\mathbf{H}_{\alpha}^{\beta}=\mathbf{T}^{-1} \mathbf{H}_{\beta^{*}}^{\beta} \mathbf{T}\right)$; (iv) calculating the stabilizer through Eqn. (3); (v) determining the degeneracy by using Lagrange Theorem (Eqn. (1)).

Because the number of transformation pathways, or deformation variants is two, a set of two order parameters $\left\{\eta_{1}, \eta_{2}\right\}$ is introduced, through which Landau free energy can be formulated in the so-called reaction coordinate [35]. By applying gradient thermodynamics [40] and Khachaturyan-Shatalov'smicroelasticity theory[41], microstructure evolution can be simulated using the phase field method [40-44]. In order to minimize the complexity and show the key features, both interfacial energy and elastic property $\left(C_{11}=3.5, C_{44}=1\right)$ are assumed to be isotropic, and the lattice parameter ratio $b / a$ is set to be 0.8 (Fig. 3).

In Landau's theory the free energy can be formulated in the so-called reaction coordinate and an order parameter along each transformation path is used to sample the lowest saddle-energy path (minimum energy pathway (MEP)) that connects the parent and product crystals. In this way, the number of order parameters is equal to the number of TPs, or the number of deformation variants, not the number of orientation variants. As a consequence, the free energy can be described by a Landau polynomial of the appropriate number of order parameters. As determined above, there are two deformation variants during a primitive-rectangle to centered-rectangle 
transformation for the given LC. A set of two order parameters $\left\{\eta_{1}, \eta_{2}\right\}$ should be introduced with $\eta_{1}=\eta_{2}=0$ representing the parent phase, while $\left\{\eta_{1}=1, \eta_{2}=0\right\}$ and $\left\{\eta_{1}=0, \eta_{2}=1\right\}$ represent the two deformation variants of the product phase. The chemical free energy density can be described by the following Landau polynomial:

$$
f=\frac{A_{2}}{2}\left(\begin{array}{c}
2 \\
1
\end{array}+\begin{array}{l}
2 \\
2
\end{array}\right)+\frac{A_{3}}{3}\left(\begin{array}{c}
3 \\
1
\end{array}+\begin{array}{l}
3 \\
2
\end{array}\right)+\frac{A_{4}}{4}\left(\begin{array}{c}
2 \\
1
\end{array}+\begin{array}{l}
2 \\
2
\end{array}\right)^{2}
$$

where the parameters $A_{2}, A_{3}$ and $A_{4}$ could be determine by fitting to ab inito calculations of the free energy along MEP in a reaction coordinate.

The deformation gradient matrices for the two deformation variants can be calculated. For convenience in elasticity, theyare described in Cartesian coordinate (i.e., $C_{0}$ in Fig. $3)$.

$$
\begin{gathered}
\mathbf{F}_{1}=\left[\begin{array}{cc}
1 & a /(2 b) \\
0 & 1
\end{array}\right] \\
\mathbf{F}_{2}=\left[\begin{array}{cc}
1 & -a /(2 b) \\
0 & 1
\end{array}\right]
\end{gathered}
$$

In general $\mathbf{F}$ may not be a symmetric matrix (Hermitian). Instead, $\mathbf{F}^{+} \mathbf{F}$ is the Hermitian that characterizes uniform lattice distortion and does not depend on OR [15, 41]. Because eigenstrainis orientation independent, it can only be a function of $\mathbf{F}^{+} \mathbf{F}$ instead of $\mathbf{F}$. One of the widely used strain formulations in continuum mechanics is the Lagrange strain,

$$
\mathbf{E}=\frac{\mathbf{F}^{T} \mathbf{F}-\mathbf{I}}{2}
$$

where $\mathbf{E}$ is the Lagrange strain and $\mathbf{I}$ is the identity. Superscript $T$ in $\mathbf{F}^{T}$ indicates the matrix transpose which is equivalent to the Hermitian operator + in real space. The resulting eigenstrains for the two deformation variants can be obtained through Eqn. (26):

$$
\begin{aligned}
& \mathbf{E}_{1}=\left[\begin{array}{cc}
0 & a /(4 b) \\
a /(4 b) & a^{2} /\left(8 b^{2}\right)
\end{array}\right] \\
& \mathbf{E}_{2}=\left[\begin{array}{cc}
0 & -a /(4 b) \\
-a /(4 b) & a^{2} /\left(8 b^{2}\right)
\end{array}\right]
\end{aligned}
$$

Thus the stress-free transformation strain can be described by:

$$
\varepsilon_{i j}^{T}(\mathbf{r})=\varepsilon_{i j}(1) \eta_{1}(\mathbf{r})+\varepsilon_{i j}(2) \eta_{2}(\mathbf{r})
$$

where $\varepsilon_{i j}(1)$ and $\varepsilon_{i j}(2)$ are the tensor forms of $\mathbf{E}_{1}$ and $\mathbf{E}_{2}$, respectively. 
The total elastic strain energy can be calculated using Khachaturyan-Shatalov's microelasticity theory[41]:

$$
E_{\text {elastic }}=\frac{1}{2} \sum_{p, q=1}^{3} f \frac{d^{3} k}{(2 \pi)^{3}} B_{p q}(\mathbf{k} /|\mathbf{k}|)\left\{\tilde{\eta}_{p}\right\}_{k}\left\{\tilde{\eta}_{q}\right\}_{k}^{*}
$$

where the integration is taken in the reciprocal space $(\mathbf{k})$. Note that $\mathbf{k}=0$ is to be excluded from the integration, which defines the principal value. Also, $\left\{\tilde{\eta}_{p}\right\}_{k}$ is the Fourier transform of $\eta$ that is a function of space. The superscript asterisk indicates complex conjugate. For a system that has fixed external boundaries, $B_{p q}(\mathbf{k} /|\mathbf{k}|)$ can be expressed as:

$$
B_{p q}(\mathbf{k} /|\mathbf{k}|)=B_{p q}(\mathbf{n})=C_{i j k l} \varepsilon_{i j}(p) \varepsilon_{k l}(q)-n_{i} \sigma_{i j}(p) \Omega_{j k} \sigma_{k l}(q) n_{l}
$$

where $\mathbf{n}=\mathbf{k} /|\mathbf{k}|$ is a unit vector in the reciprocal space, $\Omega_{i j}^{-1}=C_{i k l j} n_{k} n_{l}, \sigma_{i j}(p)=C_{i j k l} \varepsilon_{k l}(p)$.

In the simulation results (Figs. 4(a) and (b)), the shapes of deformation variant 1 and 2 are almost identical, both appearing as a thin plate with $(01)_{\alpha}$ habit determined by the elastic strain energy minimization. This is consistent with the crystallographic analysis because $(01)_{\alpha}$ is the invariant plane [16-18]. However, even though the two deformation variants are not easy to be distinguished from their morphology, they have distinctly different stress fields around them according to the phase field simulation results shown in Figs. 4(c) and (d). For example, the stress fields around the two deformation variants are symmetric to each other through a vertical (or a horizontal) mirror, which is caused by the mirror symmetry breaking along the transformation pathway. Clearly, if the orientation variant is used as the order parameter, the two equivalent pathways and different stress fields cannot be distinguished.

\subsection{HCP to $\beta_{1}$ transformation in WE54 (Mg-5wt\% Y-2wt\% Nd-2wt\% RE)}

The above theoretical formulation is equally applicable to diffusional phase transformations, if the structural part of the overall transformation is achieved under a particular lattice correspondence. In the $\mathrm{HCP} \rightarrow \beta_{1}$ transformation in magnesium alloy WE54 [45], the $\beta_{1}$ precipitate phase has a cubic structure $(F m \overline{3} m)$. According to 
experimental observations, the $\mathrm{OR}$ between $\beta_{1}$ and the $\mathrm{HCP} \mathrm{Mg}$-rich matrix is: $(1 \overline{1} 00)_{H C P} \|(\overline{1} 12)$, with $[0001]_{H C P} \|[110]_{1}$, and the following $\mathrm{LC}$ has been proposed [45]:

$$
[0001]_{H C P} \rightarrow \frac{1}{2}[110]_{1} ; \frac{2}{3}[11 \overline{2} 0]_{H C P} \rightarrow \frac{1}{2}[\overline{1} 1 \overline{1}]_{1} ; 2[1 \overline{1} 00]_{H C P} \rightarrow \frac{1}{2}[\overline{1} 13]_{1}
$$

Above $\mathrm{LC}$ is parallel to the well-known Burger correspondence during the $\mathrm{BCC}$ to HCP transformation. Note that the symmetry of occupied site in HCP indicates the point symmetry group is effectively $\overline{\mathbf{6}} \mathbf{m} 2$ (site symmetry). As result, for the calculation of transformation pathway degeneracy, symmetry groups of $\mathbf{m} \overline{3} \mathbf{m}$ and $\overline{\mathbf{6}} \mathbf{m} \mathbf{2}$ should be taken for BCC and HCP, respectively. In addition, the shuffle vector is along the direction of $[1 \overline{1} 00]_{H C P}$, being only invariant under the symmetry operations of the group of $\mathbf{m m} \mathbf{2}$, which is the stabilizer satisfying Eqn. (12).

According to Eqn. (1), six orientation variants and three deformation variants are expected from HCP to $\beta_{1}$, and the eigenstrains can be determined [46]. By ignoring the small lattice mismatch along one axis $\left([0001]_{H C P}\right)$, an invariant plane condition can be satisfied with habit planes of $\{1 \overline{100}\}$ type. The phase field simulation results with single variant particles of six orientation variants are shown in Fig. 5(a). In experimental observations, taking advantage of co-precipitation of $\beta$ 'particles as indicators of the local stress state (Fig. 5(b)), different deformation variants with the same habit plane can also be distinguished easily because of the elastic interactions between the $\beta_{1}$ and $\beta$ ' precipitates, which has been confirmed by the theoretical calculations [46].

Comparing the two sample examples above, although morphologies of precipitates in both cases are determined by the invariant plane condition, deformation variants and orientation variants are obviously different. In the first example one orientation variant corresponds to two deformation variants, while in the second example each deformation variant corresponds to two orientation variants. Therefore, the concepts of both deformation variant and orientation variant are indispensable in describing degeneracy caused by phase transformations. Besides crystal symmetry and lattice parameters of the parent and product phases, LC could be introduced as another constraint into group theory description of structural transformations, by which the 
crystal structure change and symmetry reduction can be captured. Only with a proper account of the symmetry reduction can phenomenological models be established to predict self-accommodating morphological patterns, crystallographic features and defects generated by the transformations. As demonstrated in the above examples, if the invariant plane exists, both crystallographic and energetic models provide reasonably well microstructural predictions that agree with experimental observations.

\section{Discussions}

Theoretically, transformation pathway should be a concept capturing the movements of all individual atoms and the pathway degeneracy are related to both crystal symmetry and such atomic movements. However, in order to describe the atomic movements in limited degrees of freedom, simplification assumptions have to be made. For martensitic transformations, uniform deformations have been used to describe all the atomic movements, which lead to the concept of LC (despite of its literal meaning, it actually means the atom correspondence under a uniform deformation) in martensitic crystallography [15-18]. When atomic shuffling is also involved in the structural change, additional degrees of freedom of atomic movements besides uniform deformation have to be considered in describing the TP degeneracy. In our method, both uniform deformation and atomic shuffle are considered. Note that such a description of $\mathrm{TP}$ and its degeneracy is strictly valid only in martensitic transformations. In order to generalize such descriptions to diffusional transformation, the concept of lattice site correspondence [47] has been proposed which captures the uniform deformation from one lattice to another lattice (rather than atoms). Based on such an extension, our method is equally applicable to any structural phase transition with a given lattice site correspondence.

In addition, because the TP is a concept based on atom movements, the symmetry operations affecting degeneracy should be associated only with the occupied Wyckoff position. In our group theory derivations (Section 2), the point group of a crystal structure (the point group of its space group) is used, which works for all structural phase transformations between two phases with 14 Bravais structures (Bravais lattice + monoatomic motif). However, this treatment should be improved when dealing with non-monatomic structures. For example, considering the transformation from HCP to

BCC, $\overline{\mathbf{6}} \mathbf{m} 2$ rather than $6 / \mathrm{mmm}$ should be used because it is the site symmetry for each atom in HCP (Section 3.2). As a result, it is not always correct to use the point group of a crystal structure because it may include some symmetry operations associated with non-occupied Wyckoff positions. In our current formulations, only 
one shuffle vector $\sigma$ (in Eqns. (6) \& (7)) is considered. If two or more shuffle vectors are involved during a transformation, additional constraints (i.e., invariance condition for shuffle vectors) should be add for the construction of the stabilizer.

\section{Conclusion}

In summary, a rigorous mathematical definition of deformation variants is introduced on the basis of group theory, which allows for the prediction of deformation pathway degeneracy of structural phase transformations that involve lattice deformations (including both uniform and non-uniform deformation). A general equation is derived to determine different types of degeneracy. It is shown that orientation and deformation variants are analogous but distinct concepts that can be interpreted as different ways to make a link between the two symmetry groups of the parent and product phases. For example, either the Kurdyumov-Sachs OR or Bain correspondence can be chosen as the link between the symmetry groups of FCC and BCC phases, leading to 24 orientation variants and 3 deformation variants, respectively. As a theoretical foundation of both crystallographic and energetic descriptions of structural transformations, the rigorous definition of the deformation variants introduced in this study is critical for the investigation of mechanisms that govern the formation of multi-variant domain patterns and defect structures during structural transformations. The numbers of deformation variants during forward and backward transformations determine the reversibility of the transformation and domain switching, which in turn underpins the functional properties of ferroic materials such as piezoelectricity, magnetostriction and shape memory effect.

\section{Acknowledgement}

We acknowledge the financial supports of US Department of Energy, Office of Basic Energy Sciences under Grant DE-SC0001258 (YG), the National Science Foundation under Grant No. DMR-1410322 (YW), DMR-1435483 (RS), and the Australian Research Council (ARC) Discovery Grant (JFN).

\section{Reference:}

[1] Ahart M, Somayazulu M, Cohen RE, Ganesh P, Dera P, Mao H, Hemley RJ, Ren Y, Liermann $\mathrm{P} \& \mathrm{Wu} \mathrm{Z}$. Origin of morphotropic phase boundaries in ferroelectrics. Nature 2008; 451:545.

[2] Otsuka K. \&Wayman CM. Shape Memory Materials. Cambridge: Cambridge Univ. Press; 1998.

[3] Jaffe B, Cook WR \& Jaffe H. Piezoelectric Ceramics. London:Academic Press 
Inc.; 1971.

[4] Khachaturyan AG. Ferroelectric solid solutions with morphotropic boundary: Rotational instability of polarization, metastable coexistence of phases and nanodomain adaptive states. Phil Mag 2010; 90:37.

[5] Yang S, Bao H, Zhou C, Wang Y, Ren X, Matsushita Y, Katsuya Y, Tanaka M, Kobayashi K, Song X \& Gao J. Large magnetostriction from morphotropic phase boundary in ferromagnets. Phys Rev Lett 2010; 104:197201.

[6] Van Tendeloo G \& Amelinckx S. Group-theoretical considerations concerning domain formation in ordered alloys. Acta Cryst 1974; A30:431.

[7] Wondratschek H. Splitting of Wyckoff positions (orbits). Mineral Petr 1993; 48:87.

[8] Burns G. Introduction to group theory with applications. New York: Academic; 1977.

[9] Cahn JW \&Kalonji GM. Symmetry in Solid-Solid Transformation Morphologies. In H. I. Aaronson, D.E. Laughlin, R.F. Sekerka and C.M. Wayman, editors, Proceedings of an International Conference on Solid-Solid Phase Transformations, Pittsburgh, PA, pages 3-15. AIME Press, NY, (1982).

[10] Janovec V, Hahn TH \& Klapper H. Twinning and domain structures. International Table for Crystallography (2006). Vol. D, Chapter 3.2, pp. 377-392. New York: Wiley; 2005.

[11] Sutton AP \& Balluffi RW. Overview no. 61 on geometric criteria for low interfacial energy. Acta Metall 1987; 35:2177.

[12] Bollmann W. Crystal defects and crystalline interfaces. Berlin: Springer; 1970.

[13] Bhattacharya K, Conti S, Zanzotto G \& Zimmer J. Crystal symmetry and reversibility of martensitic transformations. Nature 2004; 428:55.

[14] Otsuka K \& Ren X. Physical metallurgy of Ti-Ni-based shape memory alloys. Prog Mater Sci 2005; 50: 511.

[15] Bhattacharya K. Microstructure of martensite. New York: Oxford University Press; 2004.

[16] Wayman CM. Introduction to the crystallography of martensitic transformation. New York: Collier-Macmillan; 1964.

[17] Mackenzie JK \& Bowles JS. The crystallography of martensitic transformations II. Acta Metall 1954; 2:138.

[18] Wechsler MS, Lieberman DS \& Read TA. On the theory of the formation of martensite. Trans. AIME 1953; 197:1503.

[19] Cahn JW. The symmetry of martensites. Acta Metall 1977; 25:721.

[20] Barnighausen H. Group-subgroup relations between space groups: a useful tool in crystal chemistry. Match-Comm Math Co 1980; 9:139.

[21] Muller U. Inorganic Structural Chemistry. Chichester: Wiley; 2007.

[22] Capillas C, Perez-Mato JM, Aroyo MI. Maximal symmetry transition paths for reconstructive phase transitions. J Phys Condens Matter 2007: 19:275203.

[23] Bain E. The nature of martensite. Trans AIME 1924; 70:25.

[24] Landau L. On the theory of phase transitions. Zh. Eksp. Teor. Fiz 1937:7:19. 
[25] Li DY\& Chen LQ. Selective variant growth of coherent Ti11Ni14 precipitate in a TiNi alloy under applied stresses. Acta Mater 1997; 45:471.

[26] Zhu WJ, Chen WY \& Hsu TY. Group theory and crystallography of the martensitic transformation in a $\mathrm{Cu}-26.71 \mathrm{Zn}-4.15 \mathrm{Al}$ shape memory alloy. Acta Mater 1985; 33:2075.

[27] Zhang MX \& Kelly PM. Edge-to-edge matching and its applications: Part I. Application to the simple HCP/BCC system. Acta Mater 2005;53:1073.

[28] Burgers WG. On the process of transition of the cubic-body-centered modification into the hexagonal-close-packed modification of zirconium. Physica 1934; $1: 561$.

[29] Dahmen U. Orientation relationships in precipitation systems. Acta Metall 1982; 30:63.

[30] Christian JW. In Proceeding: ICOMAT-92, Monterey Inst. Advanced Studies, Monterey, CA, USA, 1993, pp. 233-238.

[31] Hsu TY. Martensitic transformation in Fe-Mn-Si based shape memory alloys. J Mater Sci Technol 1999; 15:111.

[32] Jian L \& Wayman CM. On the mechanism of the shape memory effect associated with $\gamma(\mathrm{fcc})$ to $\varepsilon(\mathrm{hcp})$ martensitic transformations in Fe-Mn-Si based alloys. Scripta Metall 1992; 27:279.

[33] Magee CL \& Davies RG. The structure, deformation and strength of ferrous martensites. Acta Metall 1971; 19:345.

[34] Lookman T, Shenoy SR, Rasmussen KO, Saxena A \& Bishop AR. Ferroelastic dynamics and strain compatibility . Phys Rev B 2003; 67:024114.

[35] Wang YZ \& Li J. Phase field modeling of defects and deformation. Acta Mater 2010; 58:1212.

[36] Jin YM, Artemev A \& Khachaturyan AG. Three-dimensional phase field model of low-symmetry martensitic transformation in polycrystal: simulation of $\zeta$ '2 martensite in AuCd alloys Acta Mater 2001; 49:2309.

[37] Kundin J, Raabe D, Emmerich H. A phase-field model for incoherent martensitic transformations including plastic accommodation processes in the austenite. J Mech Phys Solids 2001; 59:2082.

[38] Yeddu HK, Malik A, Agren J, Amberg G \& Borgenstam A. Three-dimensional phase-field modeling of martensitic microstructure evolution in steels. Acta Mater 2012; 60: 1538 .

[39] Schmitt R, Muller R, Kuhn C \& Urbassek HM. A phase field approach for multivariant martensitic transformations of stable and metastable phases. Arch Appl Mech (2013) 83:849.

[40] Cahn JW. \& Hilliard J. Free Energy of a Nonuniform System. I. Interfacial Free Energy. J Chem Phys1958; 28:258.

[41] Khachaturyan AG. Theory of Structural Transformations in Solids.New York: Wiley; 1983.

[42] Wang YZ \& Khachaturyan AG. Three-dimensional field model and computer modeling of martensitic transformations. Acta Mater 1997; 45:759.

[43] Chen LQ. Phase-field models for microstructure evolution. Annu Rev Mater Res 
2002; 32:113.

[44] Levitas V \& Javanbakht M. Surface tension and energy in multivariant martensitic transformations: phase-field Theory, simulations, and model of coherent Interface. Phys Rev Lett2010; 105:165701.

[45] Nie JF \& Muddle BC. Characterisation of strengthening precipitate phases in a Mg-Y-Nd alloy. Acta Mater 2000; 48:1691.

[46] Gao Y., Liu H, Shi R, Zhou N, Xu Z, Zhu YM, Nie JF \& Wang Y. Simulation study of precipitation in an Mg-Y-Nd alloy. Acta Mater 2012; 60: 4819.

[47] Hirth, JP, Aaronson HI, Rath BB \& Wayman CM. General discussion sessions of the pacific rim conference on the roles of shear and diffusion in the formation of plate-shaped transformation products. Metall Mater Trans 1994; 25:2655. 


\section{Appendix}

To make definition of stabilizer for deformation variant parallel to the definition of orientation variant, we could consider the correspondence between primitive bases of the two crystals $B_{\alpha} \rightarrow B_{\beta}$ (here $B_{\beta}$ is a particular $\beta$-basis, it is the image of $B_{\alpha}$ under the deformation, i.e. $\mathbf{a}_{i}^{\alpha} \rightarrow \mathbf{a}_{i}^{\beta}, i=1,2,3$ ). Each lattice vector (axis) in the $\alpha$-lattice is mapped to a unique lattice vector (axis) in $\beta$, and the two corresponding vectors are not necessarily parallel.

Similar to the derivations of Eqn. (9) (11), with Eqn. (8a) and (8b) taken into account, the following condition can be obtained in order to determine the stabilizer, as shown in Fig. A-1(b),

$$
\left\{\begin{array}{l}
\mathbf{h}_{\beta^{*}}^{\beta} \mathbf{P A}=\mathbf{P A h}{ }_{\alpha}^{\alpha} \\
\mathbf{h}_{\beta^{*}}^{\beta} \mathbf{P} \boldsymbol{\sigma}_{\alpha}=\mathbf{P} \boldsymbol{\sigma}_{\alpha}
\end{array}\right.
$$

PA can be considered as a general change of basis from $B_{\alpha}$ and $B_{\beta}$, which is the so-called correspondence matrix $(\mathbf{C}=\mathbf{P A})$ in martensitic crystallography [15-17]. As a result, the stabilizer that satisfies the condition for $\mathrm{LC}$ invariance is,

$$
\mathbf{J}_{D}^{\alpha / \beta}=\left\{\begin{array}{l}
\mathbf{h}^{\alpha}: \mathbf{h}_{\alpha}^{\alpha}=\mathbf{C}^{-1} \mathbf{h}_{\beta^{*}}^{\beta} \mathbf{C}, \mathbf{h}_{\beta^{*}}^{\beta} \mathbf{P} \boldsymbol{\sigma}_{\alpha}=\mathbf{P} \boldsymbol{\sigma}_{\alpha}, \\
\mathbf{h}^{\alpha} \in \mathbf{H}^{\alpha}, \mathbf{h}^{\beta} \in \mathbf{H}^{\beta}
\end{array}\right\}
$$

Where $\mathbf{P} \boldsymbol{\sigma}_{\alpha}$ (or $\boldsymbol{\sigma}_{\beta^{*}}$ ) is in fact the shuffle vector represented in $B_{\beta}$. $\boldsymbol{\sigma}_{\beta^{*}}$ has to be invariant under the symmetry operation of $\mathbf{h}_{\beta^{*}}^{\beta}$, which indicates that $\boldsymbol{\sigma}_{\beta^{*}}$ should be either along a rotation axis (if $\mathbf{h}_{\beta^{*}}^{\beta}$ is a rotation symmetry) or on the mirror plane (if $\mathbf{h}_{\beta^{*}}^{\beta}$ is a mirror symmetry).

The above equation can also be simplified if the shuffle vector is zero, which suggests a homogeneous deformation,

$$
\mathbf{J}_{D}^{\alpha / \beta}=\left\{\mathbf{h}^{\alpha}: \mathbf{h}_{\alpha}^{\alpha}=\mathbf{C}^{-1} \mathbf{h}_{\beta^{*}}^{\beta} \mathbf{C}, \mathbf{h}^{\alpha} \in \mathbf{H}^{\alpha}, \mathbf{h}^{\beta} \in \mathbf{H}^{\beta}\right\}
$$


Note that $B_{\beta^{*}}$ is a general basis rather than the special basis of $B_{\beta}$. Comparing with that in Fig. A-1(1), the operation of $\mathbf{h}^{\beta}$ in Fig. A-1(b) can be described in any basis as convenient, which will facilitate greatly the mathematical procedure to determine the number of deformation variants. 


\section{Figure captions:}

Fig. 1. Diagrammatic illustration of the relationships among operator $\hat{\mathbf{T}}$, symmetry operations $\left(\mathbf{h}^{\alpha}, \mathbf{h}^{\beta}\right)$ and lattice vectors $\left(\mathbf{r}^{\alpha}, \mathbf{r}^{\beta}, \mathbf{s}^{\alpha}, \mathbf{s}^{\beta}\right)$ in respective lattices. Different choice of $\hat{\mathbf{T}}$ could lead to different degeneracy. (a) $\hat{\mathbf{T}}$ is a generic operator capturing the relationships (e.g., change of basis, lattice distortion, or the combination of the two) between two crystals of $\alpha$ and $\beta$; (b) $\hat{\mathbf{T}}$ is realized by $\hat{\mathbf{P}}$ that is a passive operator describing change of basis from $B_{\alpha}$ to $B_{\beta}$ (e.g., a rotation of one lattice relative to the other), so that the circuit shows the condition for determining orientation relationship (OR) degeneracy; (c) $\hat{\mathbf{T}}$ is realized by $\mathbf{A}$ and $\boldsymbol{\sigma}$ that describe an active operation (e.g., transformation or deformation) bringing $\alpha$ lattice to $\beta$ lattice under a given lattice correspondence (LC), so that the circuit shows the condition for determining transformation pathway (TP) degeneracy.

Fig. 2. Schematic illustration of pathway degeneracies for transformations among FCC, BCC and HCP.

Fig. 3. Unit cells for a primitive-rectangle to centered-rectangle transformation with lattice parameters, OR, and LC indicated. Note the two deformation variants for the same orientation (the bottom row).

Fig. 4. Difference between two deformation variants demonstrated by phase field simulations: (a) variant 1; (b) variant 2 ; (c) $\sigma_{11}$ around deformation variant 1 ; (d) $\sigma_{11}$ around deformation variant 2 (normalized by $100 \times \mathrm{C}_{44}$ ).

Fig. 5. Simulation and experimental results of three deformation variants and six orientation variants of $\beta_{1}$ phase: (a) six $\beta_{1}$ orientation variants formed by three deformation variants. In each row the two orientation variants are formed by the same deformation variant; (b) TEM image of $\beta_{1}$ precipitates[46], from which we can see that $\beta$ particles are attached only to fixed location of the $\beta_{1}$ particles (as indicated by the red circles in (a)) due to their elastic interactions.

Fig. A-1. Diagrammatic illustration of the relationships among operator $\hat{\mathbf{T}}$ (a combination of basis change and lattice distortion), symmetry operations $\left(\mathbf{h}^{\alpha}, \mathbf{h}^{\beta}\right)$ and 
lattice vectors $\left(\mathbf{r}^{\alpha}, \mathbf{r}^{\beta}, \mathbf{s}^{\alpha}, \mathbf{s}^{\beta}\right)$ in respective lattices: (a) $\hat{\mathbf{T}}$ provides a relationship between the bases of $B_{\alpha}$ and a special $B_{\beta}$; (b) $\hat{\mathbf{T}}$ provides a relationship between the bases of $B_{\alpha}$ and a general $B_{\beta}$, so that the circuit shows the condition for determining transformation pathway (TP) degeneracy in a general way. 
Figure 1

(a)

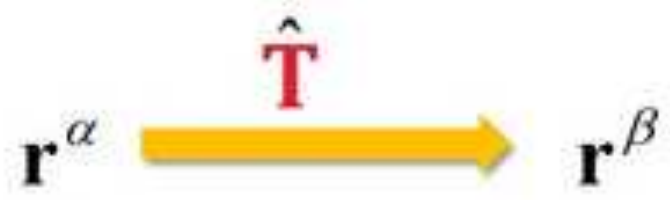

$\mathbf{h}^{\alpha}=\hat{\mathbf{T}}^{-1} \mathbf{h}^{\beta} \hat{\mathbf{T}}$

$\mathbf{h}^{\beta}$

(b)

$\hat{\mathbf{P}}$

$\mathbf{r}_{\alpha}$

$\mathbf{r}_{\beta}$

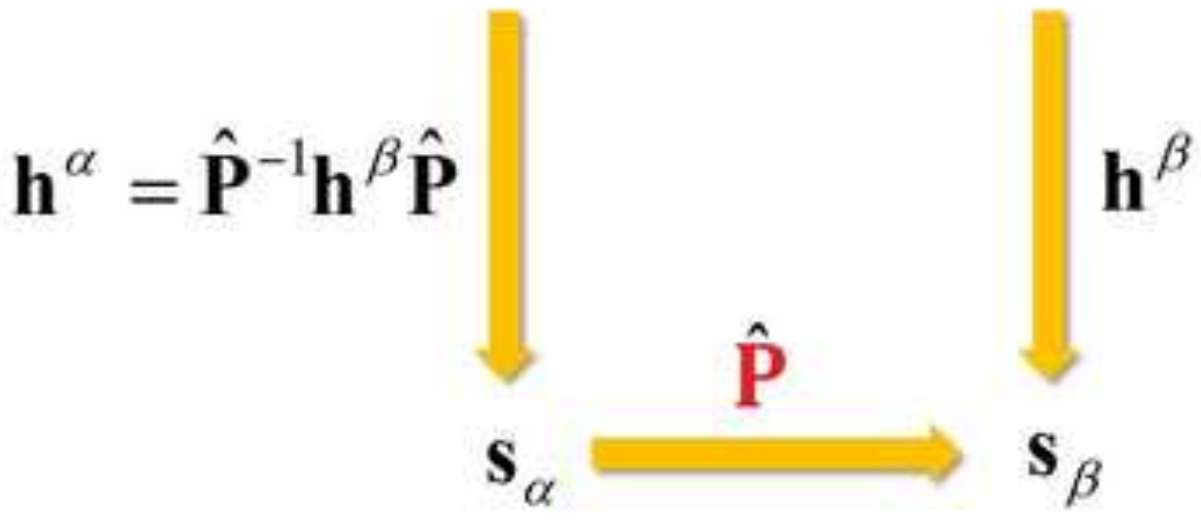

(c)

$\hat{\mathrm{T}} \Rightarrow \mathbf{A r _ { \alpha } ^ { \alpha }}+\boldsymbol{\sigma}_{\alpha}$

$$
\mathbf{r}_{\alpha}^{\alpha}
$$

$\mathbf{r}_{\alpha}^{\beta}$

$\mathbf{h}^{\alpha}=\hat{\mathbf{T}}^{-1} \mathbf{h}^{\beta} \hat{\mathbf{T}}$
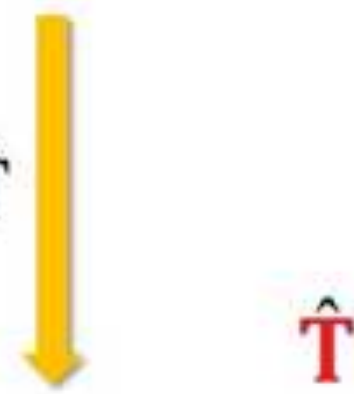

$\mathbf{s}_{\alpha}^{\alpha}$

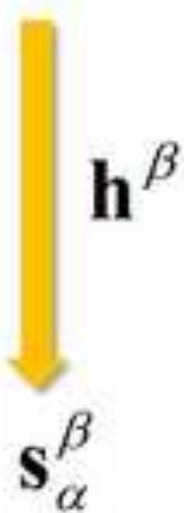




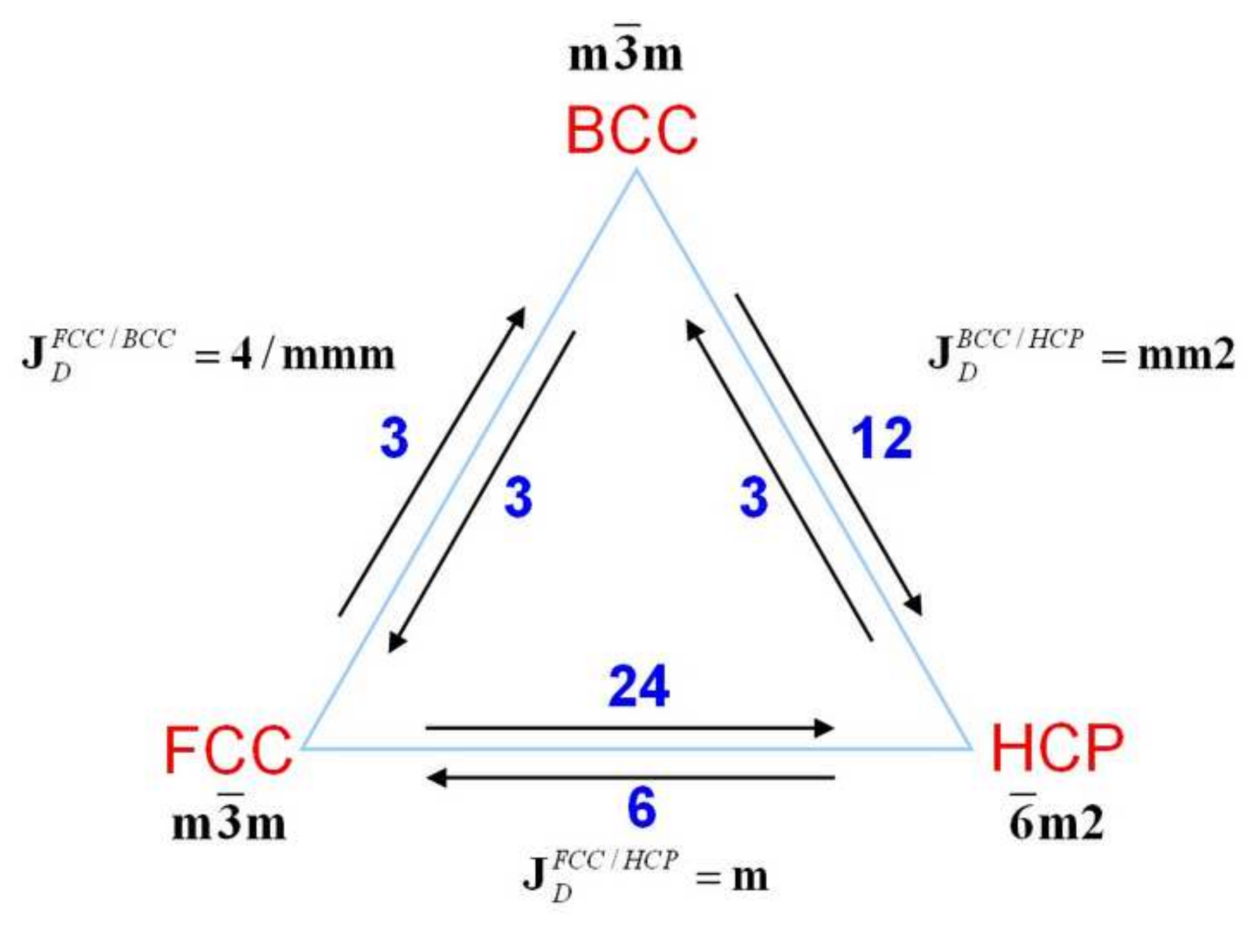

m $\overline{3}$ nn 

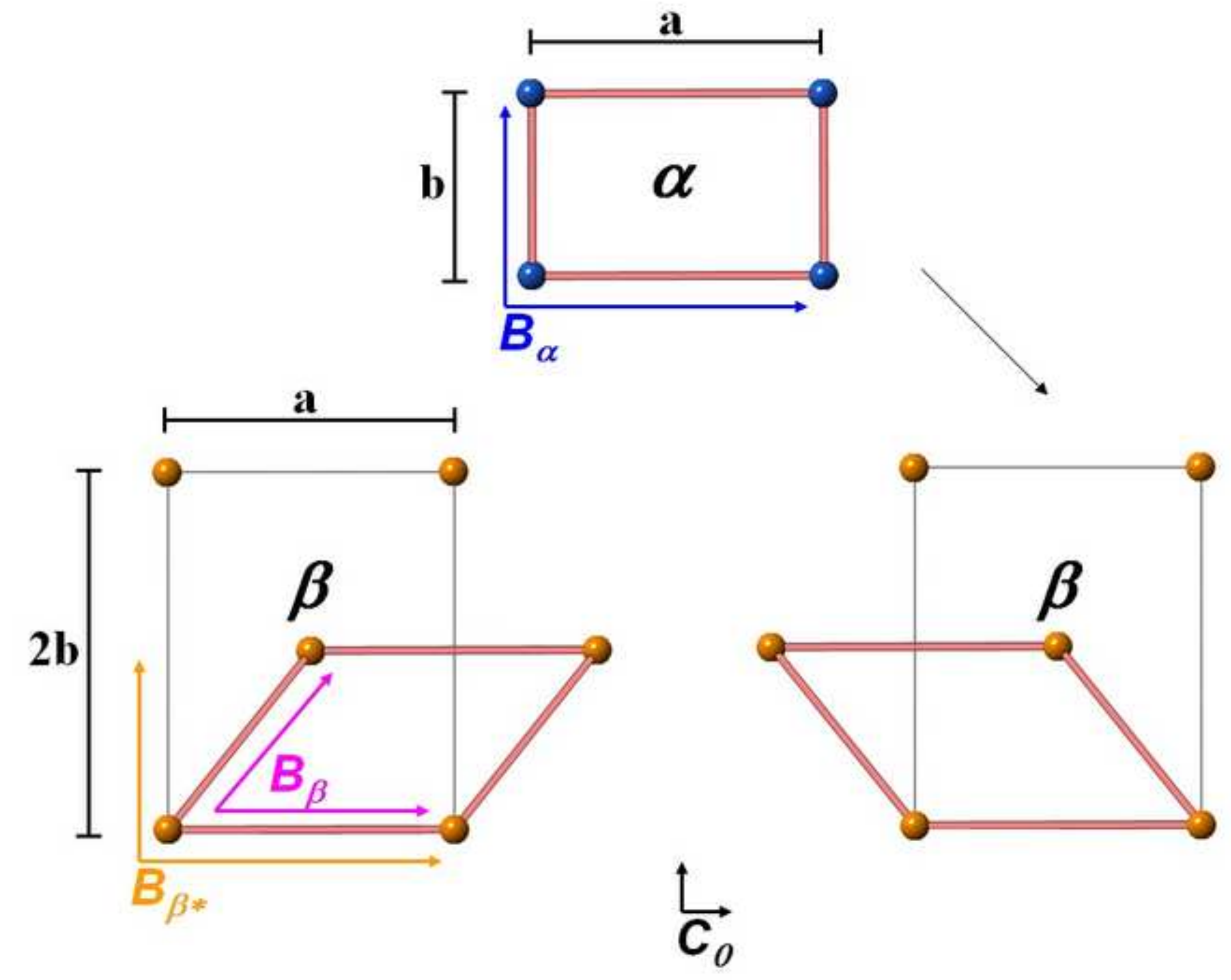
(a)

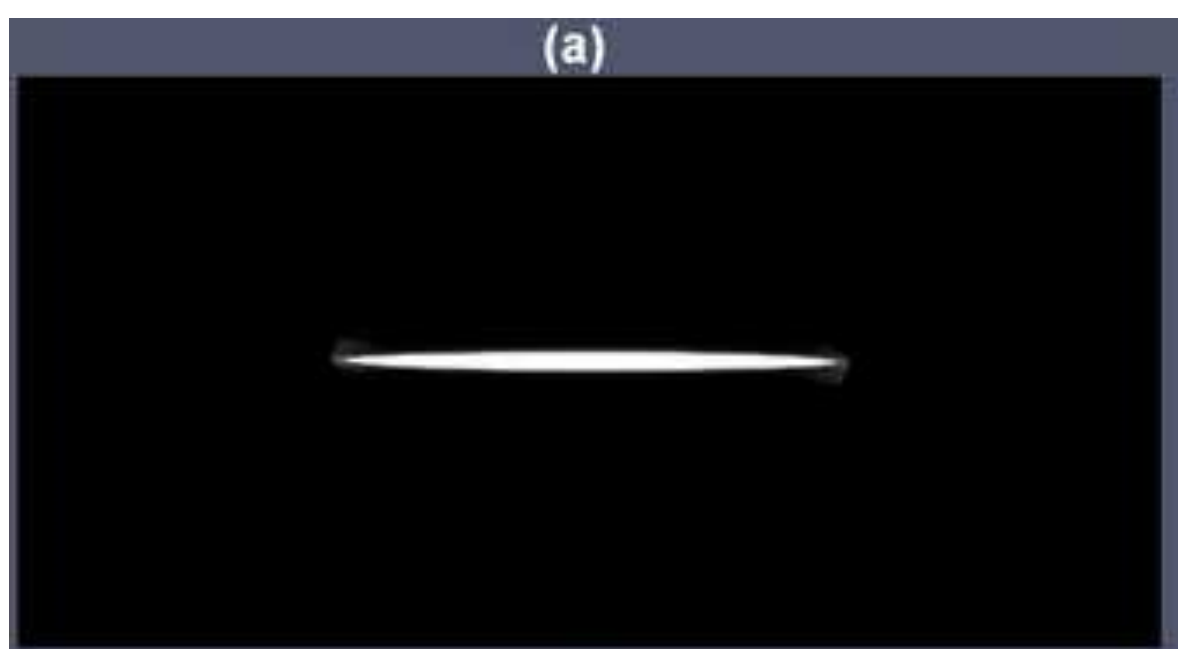

(c) (b)

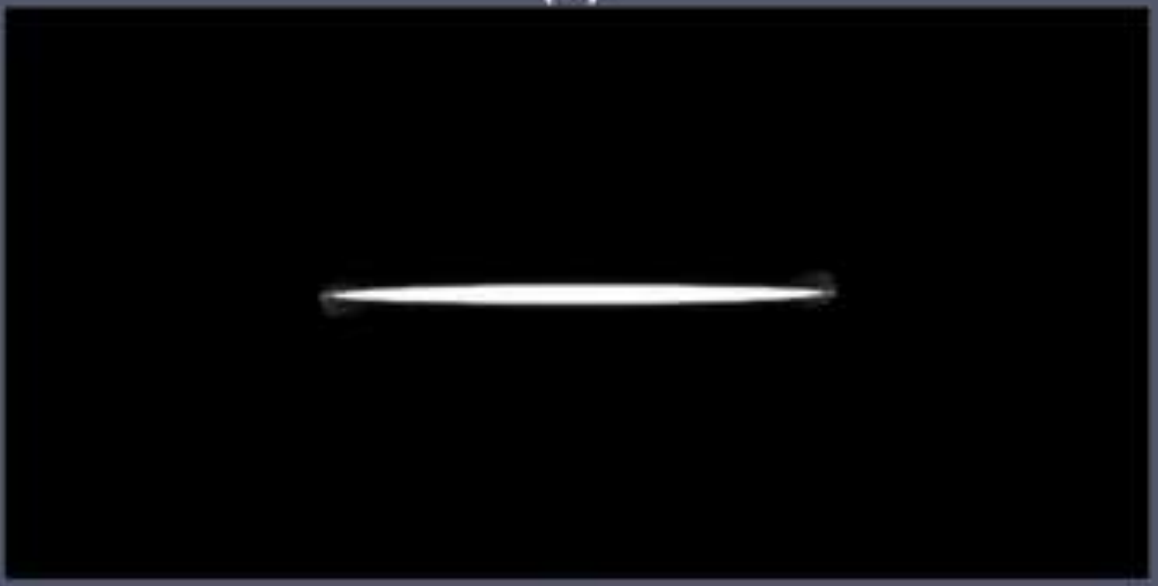

(d)

13.75

10

0

$-10$

$-15.39$

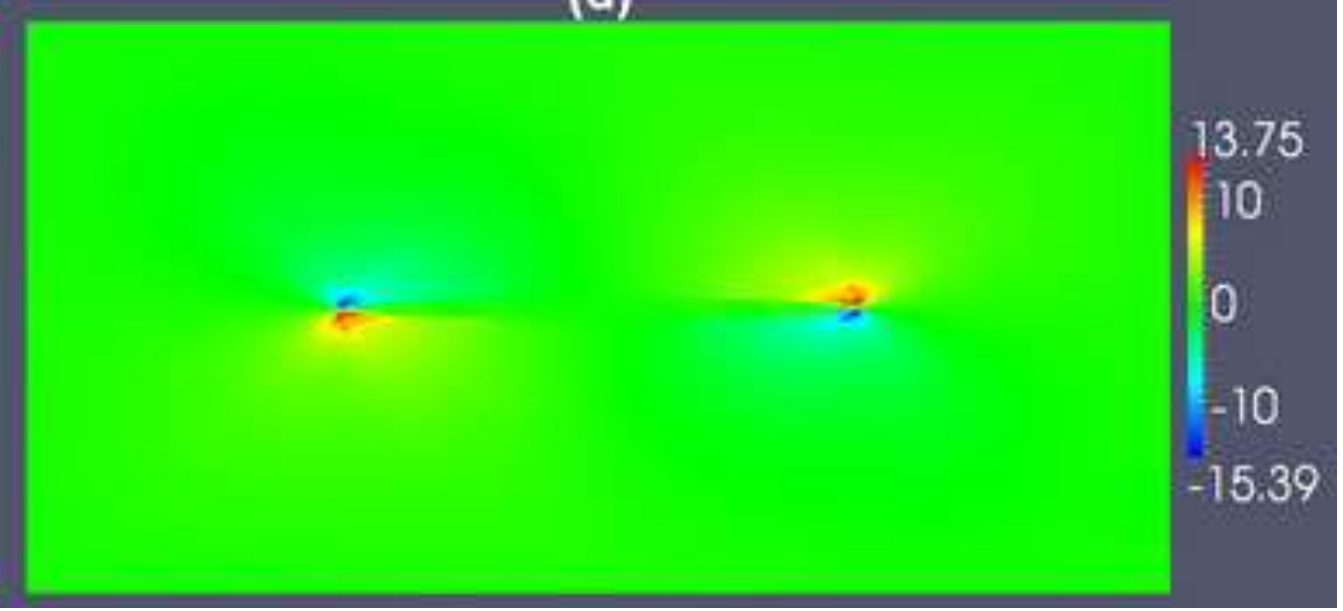

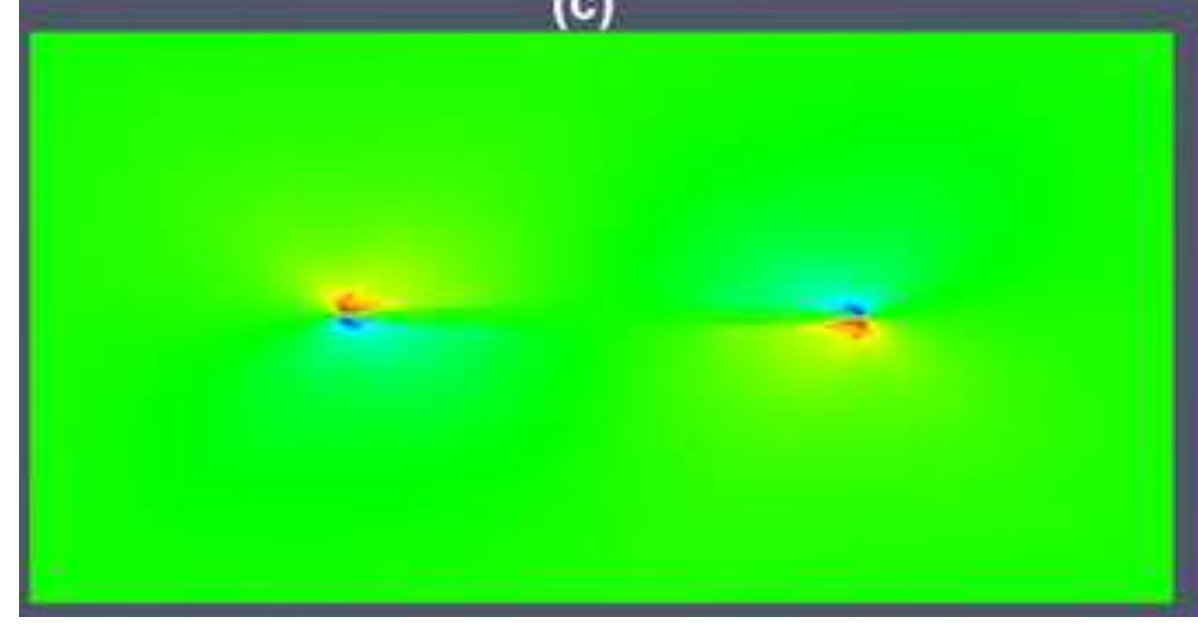


(a)
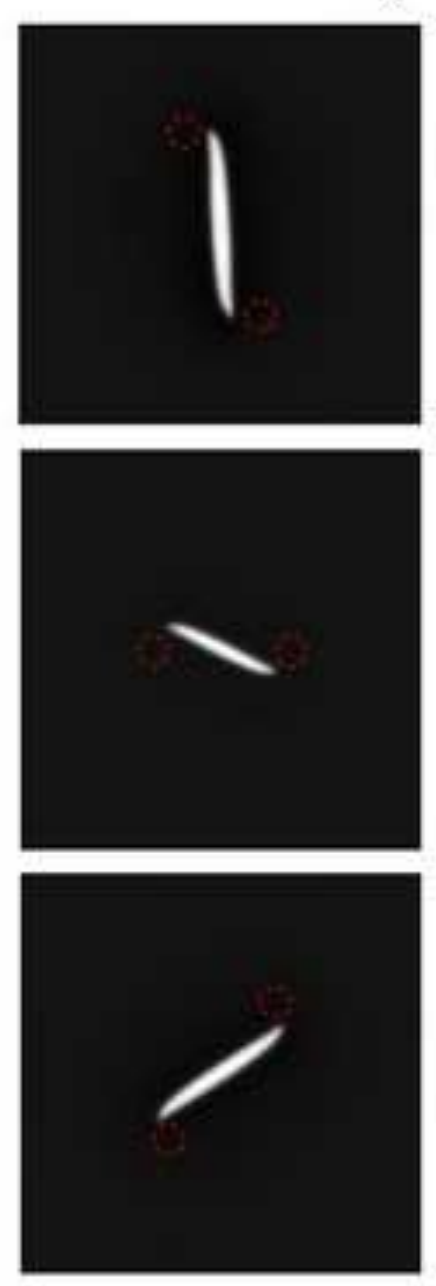
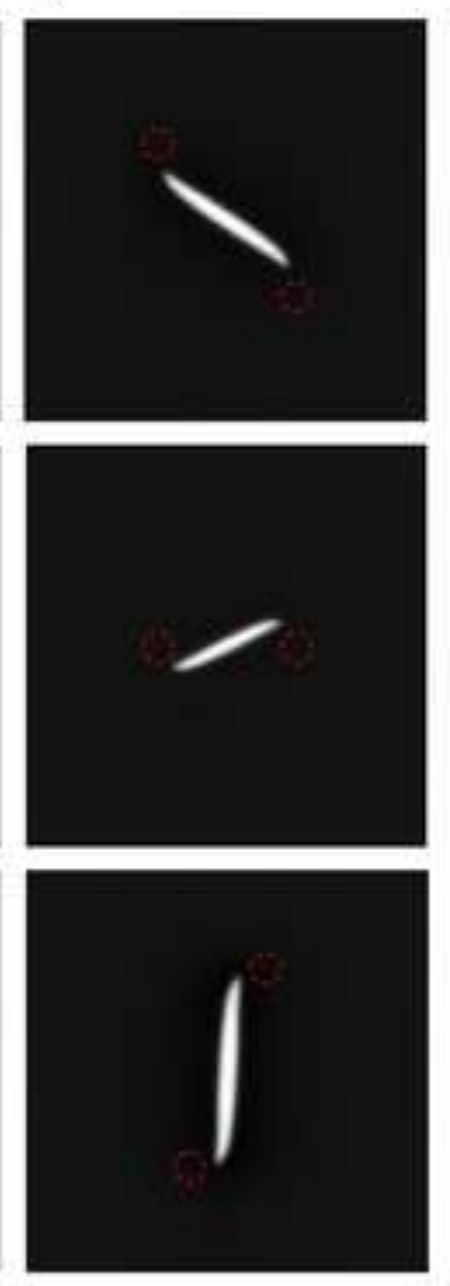

(b)

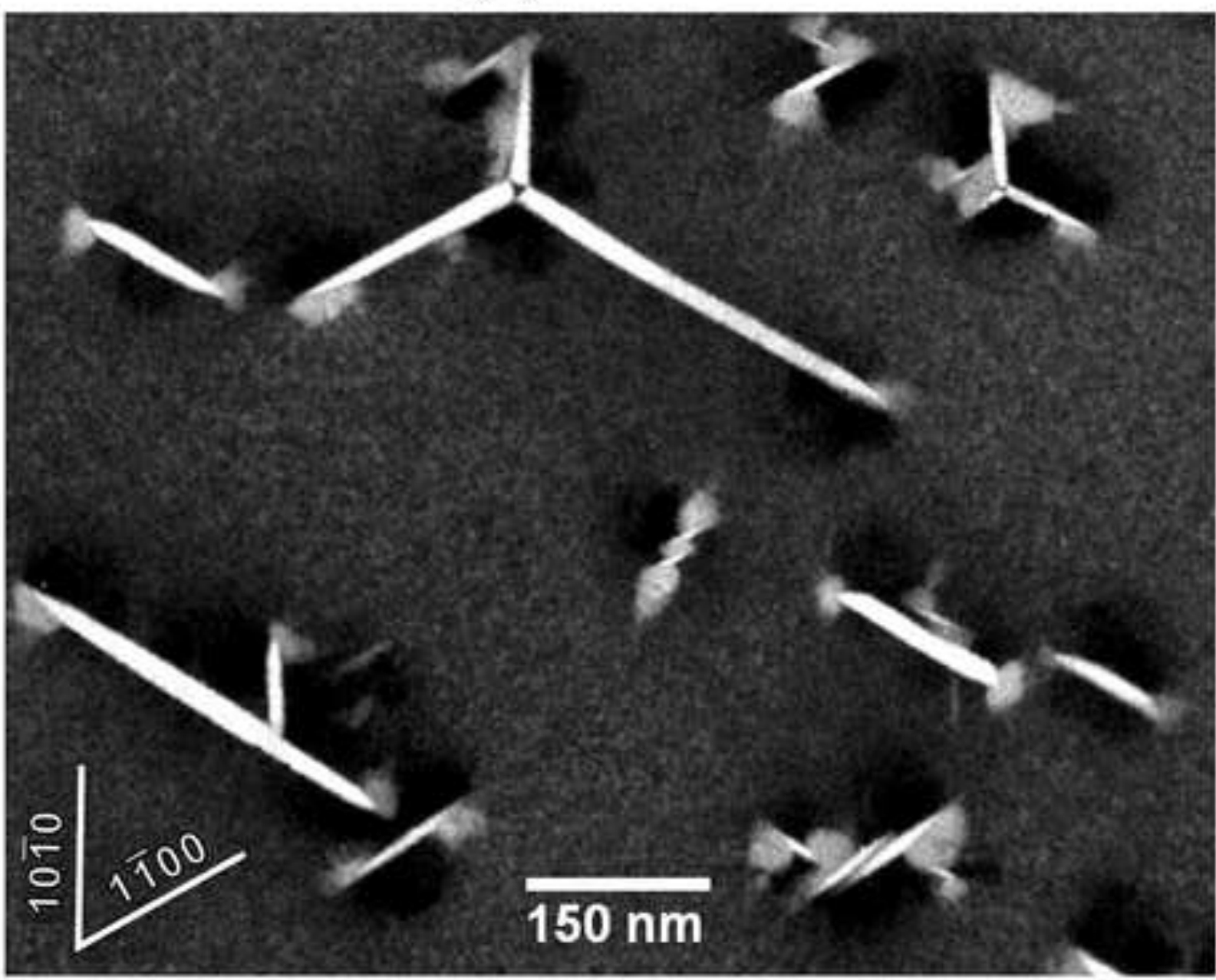

$150 \mathrm{~nm}$ 
(a)

$\hat{\mathbf{T}} \Rightarrow \mathbf{P A}=\mathbf{I}$

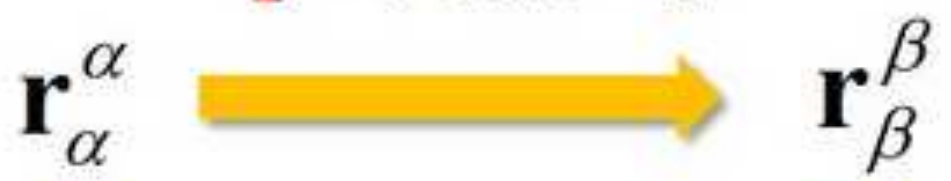

$\mathbf{h}^{\alpha}=\hat{\mathbf{T}}^{-1} \mathbf{h}^{\beta} \hat{\mathbf{T}}$

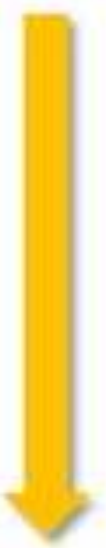

$\mathbf{s}_{\alpha}^{\alpha}$

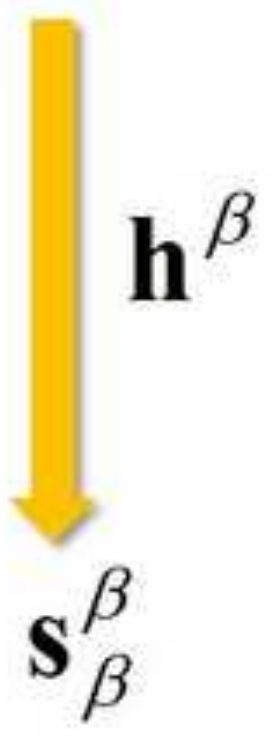

(b)

$\hat{\mathbf{T}} \Rightarrow \mathbf{P}\left(\mathbf{A r}_{\alpha}^{\alpha}+\boldsymbol{\sigma}_{\alpha}\right)$

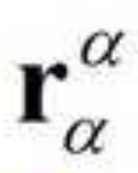

$\mathbf{r}_{\beta^{*}}^{\beta}$

$\mathbf{h}^{\alpha}=\hat{\mathbf{T}}^{-1} \mathbf{h}^{\beta} \hat{\mathbf{T}}$
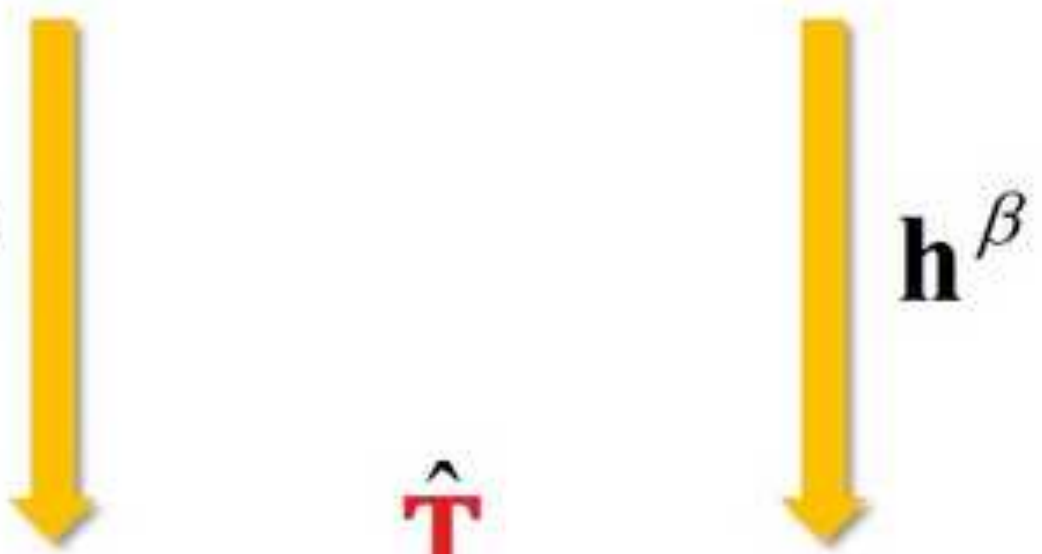

$\mathbf{S}_{\alpha}^{\alpha}$
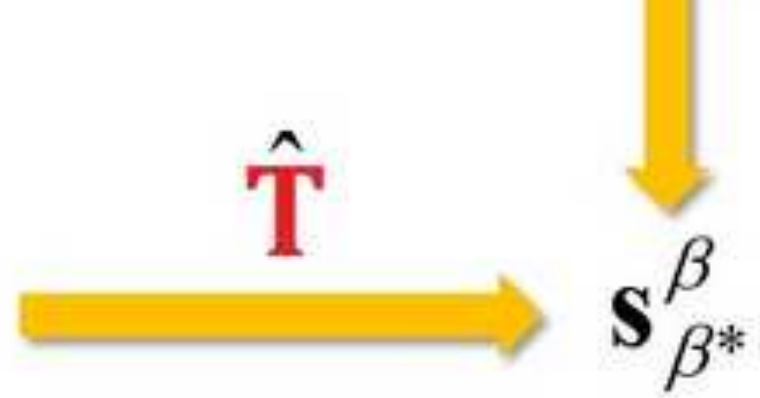


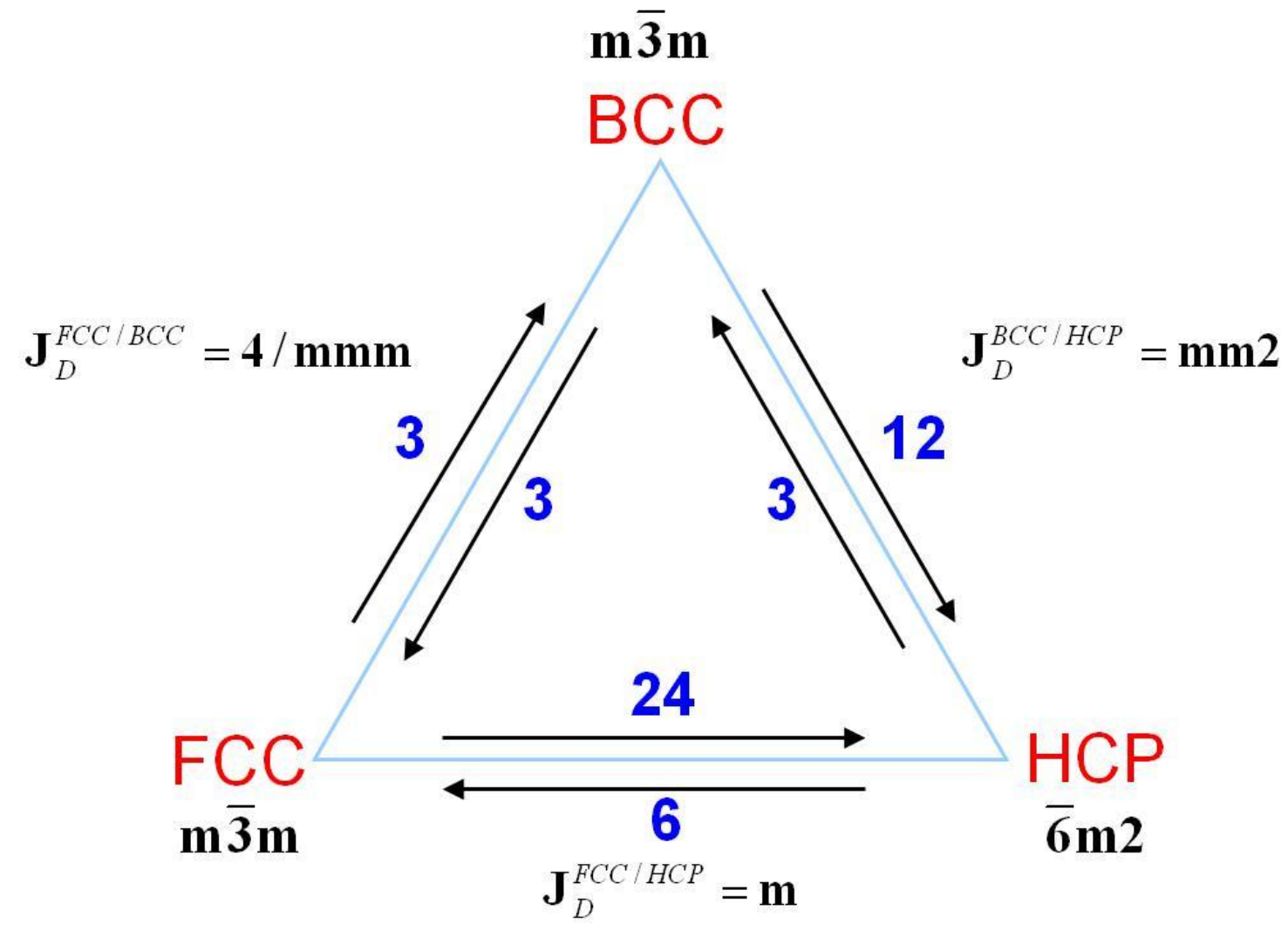

Schematic illustrations of symmetry breaking and pathway degeneracy during structural phase transformations among FCC, BCC and HCP crystals. 